\title{
25 Research Sourere \\ Effects of Distinct n-6 to n-3 Polyunsaturated Fatty Acid Rations on Insulin Resistant and AD-like Phenotypes in High-Fat Diets-Fed APP/PS1 Transgenic Mice
}

\section{Xiaojun Ma}

Capital Medical University

Yujie Guo

Capital Medical University

Pengfei Li

Capital Medical University

Jingjing Xu

Capital University of Medical Sciences: Capital Medical University

Shengqi Dong

Capital Medical University

\section{Yanyan Gao}

Capital Medical University

Nicholas Van Halm Lutterodt

Capital Medical University

Linhong Yuan ( $\nabla$ ylhmedu@126.com )

Capital Medical University https://orcid.org/0000-0001-5169-2527

\section{Research Article}

Keywords: High-fat diet, insulin resistance, n-6/n-3 PUFA ratio, AD-like pathology, APP/PS1 mice

Posted Date: June 8th, 2021

DOI: https://doi.org/10.21203/rs.3.rs-582409/v1

License: (c) (1) This work is licensed under a Creative Commons Attribution 4.0 International License. Read Full License 


\section{Abstract}

Background: Type 2 diabetes mellitus (T2DM) and Alzheimer's disease (AD) are two prevalent diseases with comparable pathophysiological features and genetic predisposition. Polyunsaturated fatty acids (PUFAs) are essential in maintaining normal brain function. However, little is known about the impact of dietary n-6/n-3 PUFA ratio on AD-like pathology, especially in high-fat diet (HFD)-fed AD model mice.

Methods: In the present study, the APP/PS1 mice were treated with 60\% HFD for 3.5 months to induced insulin resistance. After that, 45\% HFD with different n-6/n-3 PUFA ratios (n-6/n-3=1:1, 5:1 or 16:1) was applied for additional 3.5 months treatment. Following the dietary intervention, the behavior of mice was observed using the Water maze. Following behavioral testing, the animals were euthanized, and serum and tissue samples were collected for biochemical, histological and pathological analyses and evaluation. Cortical fatty acid profile was measured by gas chromatography. Western Blot and immunohistochemistry methods were used to detect protein expression of molecules related to AD pathology and insulin signaling pathway(s) in the brain sample tissues. Immunofluorescence assay was used to uncover the expression and migration of NF-KB in the cortex. qPCR method was applied to determine the gene expression of cortical pro-inflammatory cytokines.

Results: HFD caused insulin resistance, increased serum IL-6 and TNF-a level, elevated cortical soluble $A \beta_{1-40}, A \beta_{1-42}$ content, and increased brain n-6/n-3 PUFAs ratio in APP/PS1 mice. Increased APP and BACE1 protein expression and $p$-IR/IR ratio, but decreased pro-inflammatory cytokines mRNA expression was observed in the cortex from 60\% HFD-fed APP/PS1 mice. N-3 PUFAs rich diet $(n-6 / n-3=1: 1)$ relieved insulin resistance and hyperlipidemia induced by $60 \%$ HFD. Cortical soluble $A \beta_{1-40}$ and $A \beta_{1-42}$ contents, the expression of cortical APP, GLUT3, insulin metabolism related molecules, and NF-KB pathway downstream pro-inflammatory cytokines showed a dietary n-6/n-3 PUFAs ratio-dependent way, indicating that dietary $n-6 / n-3$ PUFA ratio plays a critical role in modifying the responses of serum inflammatory cytokine, AD pathology, cortical n-6/n-3 PUFAs ratio, insulin signaling and neuroinflammation to HFD treatment.

Conclusion: Dietary n-6/n-3 PUFA ratio play an important role in modifying AD pathophysiology, insulin signaling pathway, and neuro-inflammation response to high fat diet treatment in brain.

\section{Background}

With accelerated aging of the world's population, dementia is undoubtedly becoming a growing global public health concern. Alzheimer's disease (AD) is the leading cause, accounting for about $70 \%$ of all dementia cases [1]. Although, tremendous efforts have been made in understanding the pathogenesis of $A D$, unfortunately, there still remains no available definitive or effective disease-modifying treatment approaches. Therefore, targeting $A D$ at an earlier stage and mitigating or delaying the disease process seems to be promising strategies to help prevent $A D$. 
Metabolic disturbances in the brain are reported to be principally involved in the onset and progression of $A D$ [2]. Recent population-based epidemiological studies indicate that diabetic patient at an increased risk of developing $A D$ [3]. Furthermore, about $80 \%$ of $A D$ patients are also diagnosed with diabetes or glucose intolerance [4], and significantly reduced quantities of both insulin and insulin-like growth factor 1 (IGF-1) were observed in AD subjects' postmortem brains [5], suggesting that diabetes may play a causative role in the development of $A D$ pathogenesis. Altered insulin signaling pathway in type 2 diabetes mellitus (T2DM) patients, including raised fasting blood glucose, glucose intolerance and insulin resistance, might form a coordinated link between $A D$ and T2DM, and it was also speculated that $A D$ could be a third form of diabetes (type $\otimes$ diabetes) [6]. Since $A D$ and T2DM are the predominant underlying health conditions affecting the aging population, the largest volume of $A D$ and T2DM can undoubtedly be anticipated, in low- and middle-income countries [7]. However, to date, the underlying mechanisms involved in diabetesinduced cognitive impairment and pathogenesis are not fully elucidated. It is of critical significance to illuminate the underlying molecular interactions between diabetes and $A D$, which would enable strategies for early prevention and/or intervention of dementia.

The long-chain polyunsaturated fatty acids (LCPUFAs) are important components of neuronal membranes, and essential for neuronal membrane integrity and function. The n-3 PUFAs, especially docosahexaenoic acid (DHA), are the major constituting acids of neurological and retinal membranes [8]. Data from population-based epidemiological studies consistently showed that the consumption of n-3 PUFAs-rich diets potentially reduces the risk of developing neurological disorders [9]. Reduced or depletion of n-3 PUFAs levels from erythrocyte membranes was observed in AD patients [10]. A retrospective study including 1,188 elderly American subjects reported that low levels of circulating DHA may pose a significant risk in the development of Alzheimer's dementia [11]. On the contrary, n-6 PUFAs and their conversion products favor immune and inflammatory reactions, which can contribute to the development of dementia. High erythrocyte total n-6 PUFA concentration was also suggested to negatively correlate with cognitive function [12]. Furthermore, diets enriched in n-6 PUFAs have also been reported to increase the production of arachidonic acid (ARA), causing islet inflammatory damages, ultimately contributing to insulin resistance and diabetes, which were proposed risk factors for dementia in aging subjects [13]. All these data demonstrate the important role of $n-6$ and $n-3$ PUFAs alterations in influencing the pathological process of dementia. A balanced intake of both n- 6 and n-3 PUFA rations may be essential for reducing oxidative stress, inflammatory status, the incidence of diabetes, and thereby, decreasing the prevalence of AD. Data from the 2002 survey on nutrition and health status of Chinese residents reported that the values of $n-6$ to $n-3$ polyunsaturated fatty acid ratios $(n-6 / n-3)$ in urban and rural residents were 7.6 and 8.0 respectively [14], which are much higher than the recommended Chinese dietary $n-6 / n-3$ ratio reference (4-6:1). In western countries, the dietary $n-6 / n-3$ PUFA ratio was reported as 20:1, or even higher [15]. Currently, to our knowledge, few studies have explored the associated impact of dietary n-6/n-3 PUFA ratio on AD-like pathology and insulin metabolism in the brain, especially in HFD-treated AD model mice. The present study mainly focuses on clarifying the impacts of dietary n-3/n-6 PUFA ratio on AD-like and insulin resistant phenotypes in HFDfed $A D$ model mice. Our data highlight the significant role of dietary $n-6 / n-3$ PUFAs ratio on affecting the 
etiology of $A D$, as well as revealing the fundamental pathophysiological mechanisms of insulin resistant related dementia.

\section{Methods}

\section{Animals and diet}

A total of 36 male APP swe $/ P S 1_{\mathrm{dEg}}$ transgenic mice and 6 sex-matched $\mathrm{C} 57 \mathrm{BL} / 6 \mathrm{~J}$ control mice (all at 4 weeks old) were purchased from the experimental animal center of Capital Medical University. The mice were maintained on a 12:12 light-dark cycle and allowed access to water and food ad libitum. Experiment protocols were licensed according to Capital Medical University Animal Care and Use Committee regulations and associated guidelines (AEEl-2019-071). All diets were purchased from SYSE Bio-tech. Co. LTD, Changzhou, China. The normal diet (ND) and HFD differ in the composition of fat added to the diet. Full details of the diet are provided in Table 1. 
Table 1

Composition of experimental diets.

\begin{tabular}{|c|c|c|c|c|c|}
\hline $\begin{array}{l}\text { Nutrients in } \\
\operatorname{diet}(\mathrm{g} / \mathrm{kg})\end{array}$ & $\begin{array}{l}\text { Normal control } \\
\text { diet }\end{array}$ & $60 \%$ HFD & 45\% HFD & 45\% HFD-N3 & 45\% HFD-N6 \\
\hline Protein & 192 & 260 & 240 & 240 & 240 \\
\hline Carbohydrate & 673 & 260 & 410 & 410 & 410 \\
\hline Fat & 350 & 350 & 240 & 240 & 240 \\
\hline kcal & 3850 & 5240 & 4730 & 4730 & 4730 \\
\hline \multicolumn{6}{|l|}{ Ingredient } \\
\hline Casein & 200 & 200 & 200 & 200 & 200 \\
\hline L-Cystine & 3 & 3 & 3 & 3 & 3 \\
\hline Corn Starch & 315 & 0 & 72.8 & 72.8 & 72.8 \\
\hline Maltodextrin & 35 & 125 & 100 & 100 & 100 \\
\hline Sucrose & 350 & 68.8 & 172.8 & 172.8 & 172.8 \\
\hline Cellulose & 50 & 50 & 50 & 50 & 50 \\
\hline Soybean oil & 9.1 & 25 & 34.3 & 0 & 21 \\
\hline Lard & 33.5 & 245 & 158 & 165.5 & 153.5 \\
\hline Perilla oil & 0 & 0 & 0 & 37 & 0 \\
\hline Flaxseed oil & 2.4 & 0 & 10.2 & 0 & 1.9 \\
\hline Corn oil & 0 & 0 & 0 & 0 & 26.1 \\
\hline Mineral Mix & 45 & 45 & 45 & 45 & 45 \\
\hline Vitamin Mix & 10 & 10 & 10 & 10 & 10 \\
\hline \multicolumn{6}{|l|}{ Fatty acid } \\
\hline SFA & 12.4 & 105.4 & 71.6 & 71.3 & 70.6 \\
\hline MUFA & 17.1 & 112.3 & 78.4 & 76.9 & 79.4 \\
\hline PUFA & 12.4 & 52.5 & 52.6 & 54.5 & 52.5 \\
\hline n-6/n-3 PUFA & 5.0 & 20.0 & 5.0 & 1.1 & 16.0 \\
\hline
\end{tabular}


According to baseline fasting blood glucose level, 36 male APP/PS1 mice were randomly split into 2 treatment groups: (1) ND group $(n=6)$ : the mice were fed with a standard diet, in which $10 \% \mathrm{kcal}$ are derived from fat chow (10 kcal\%, Product code: D18091704); (2) HFD group $(n=30)$ : in this group, the mice were fed with high-fat diet, in which $60 \% \mathrm{kcal}$ are derived from fat (60 kcal\%, Product code: D12492). Sucrose content per gram was equivalent between the two diets. Diet intakes of animals were monitored every 3 days, and body weights were assessed weekly. Plasma glucose levels of APP/PS1 mice treated with 60\% HFD were monitored monthly. After 60\% HFD dietary intervention for 3.5 months, glucose tolerance test (GTT) was performed to assess insulin resistance phenotype. Any mouse that did not show an increase in plasma glucose in comparison with control mice was excluded from the study. At the age of 4.5 months, the mice with increased fasting glucose level were further randomly divided into 4 subgroups (at least 6 mice for each group): (1) the high n-6/n-3 PUFA ratio diet group: the mice were provided with 45\% HFD with $n-6 / n-3=16: 1$ (Product code: D18091701); (2) the medium n-6/n-3 PUFA ratio diet group: the mice were treated with $45 \%$ HFD with $n-6 / n-3=5: 1$ (Product code: D8091703); (3) the low $n-6 / n-3$ PUFA ratio diet group: the mice were treated with $45 \%$ HFD with $n-6 / n-3=1: 1$ (Product code: D18091702); (4) the $60 \%$ HFD group: the mice were fed with their original $60 \%$ HFD diet. The control APP/PS1 mice and C57BL/6J mice were maintained on their original control diets. After 3.5 months under these dietary regimens, the mice were used for GTT and behavioral measurement. The whole schematic diagram of animal dietary intervention is shown in Supplementary Fig. 1.

\section{Fasting blood glucose levels and GTT}

Blood glucose levels were measured per month, using a standard glucometer (Accu-Chek, Roche) with blood obtained from the tail tip. GTT were performed at the time point of $60 \%$ HFD intervention for 14 weeks, and the end of the experiment.

\section{Behavioral testing}

At the end of the dietary intervention, the spatial learning and memory abilities of animals were analyzed by Morris water maze as described previously [16]. The escape latency, path length travelled to arrive at the platform, average swim speed, and platform site crossings were recorded with a video tracking system (Water Maze 2.6 Institute of Materia, Chinese Academy of Medical Sciences DMS-2, Beijing, China).

\section{Tissue preparation}

Following behavioral testing, all mice were euthanized and sacrificed. Their brains were removed and separated along the midsagittal sulcus. Half of each brain was immediately placed in a cold saline solution and then the cortical and hippocampal regions were dissected and stored at $-80^{\circ} \mathrm{C}$ until used for biochemical measurements. The other half side of the brain was used for the histological studies. Other tissue samples including liver, muscle and adipose tissue were frozen at $-80^{\circ} \mathrm{C}$ for biochemical assays or fixed in formalin for histological analyses. 


\section{Histology, immunofluorescent and immunohistochemical assay}

The liver samples were embedded in $10 \%$ formaldehyde buffered solution, subsequently embedded in paraffin blocks, and sectioned into slices of $5 \mu \mathrm{m}$, and then stained with hematoxylin-eosin (HE).

Specimens were examined under a light microscope. Beta-amyloid plaque in the cortex and hippocampus was measured by using Congo red (KeyGEN BioTECH, Nanjing, China) staining kit.

Immunofluorescent (IF) analysis was applied to detect the expression and activation of cortical nuclear factor kappa-B p65 (NF-kB p65). The 4',6-diamidino-2-phenylindole (DAPI) glows blue by UV excitation wavelength 330-380 nm and emission wavelength $420 \mathrm{~nm}$, and NF-KB p65 glows red by excitation wavelength $510-560 \mathrm{~nm}$ and emission wavelength $590 \mathrm{~nm}$ by using laser scanning confocal microscope (Leica, Solms, Germany).

Immunohistochemistry (IHC) assay was applied for measuring glucose transporters (GLUTs) in tissues. Briefly, brain, liver, skeletal muscle and omental fat tissue samples were fixed for 48 hours, embedded in paraffin, and then sectioned into slices of $5 \mu \mathrm{m}$. IHC was performed on paraffin-embedded sections using antibodies directed against GLUT2, (1:100 dilution, Cell Signaling Technology, USA), GLUT3, (1:100 dilution, Cell Signaling Technology, USA), and GLUT4 (1:100 dilution, Proteintech, USA). Secondary DAB antibody was used for positive detection. Then, the section was observed and photographed by electric microscope (OLYMPUS BX61).

\section{Cortical fatty acids profile measurement}

The fatty acid concentration in the cortexes was determined according to the method described previously [17]. Fatty acid compositions of 3-4 brains per group were reported, and the fatty acids contents were expressed as $\mathrm{g} / 100 \mathrm{~g}$ total fatty acids.

\section{ELISA and biochemical assay}

Serum TNF-a and IL-6 contents were measured using commercial ELISA kits (Immunoway, Suzhou, China) according to the manufacturer's instruction. Total cholesterol (TC) and low-density lipoprotein cholesterol (LDL-c) level in the cortex was determined by ELISA kit (mlbio, Shanghai, China) according to manufacturer's instructions. Cortical insulin level was determined by ELISA kit (Elabscience Biotechnology Co., Ltd, Wuhan, China). The levels of soluble and aggregated $A \beta_{1-40}$ and $A \beta_{1-42}$ were measured by human $\beta$-Amyloid ELISA kits (Wako, Osaka, Japan) according to the manufacturer's instructions. Three independent measurements were performed for each sample.

\section{Western blotting}

A total of $20 \mu \mathrm{g}$ of protein lysis extracts were electrophoresed on Tris-tricine gradient gels as described previously [18]. And primary antibodies used in western blotting were shown in Supplementary Table 1. RNA extraction and qRT-PCR 
Cortical total RNA was extracted using Ultrapure RNA Kit (Cwbio, Jiangsu, China) according to the manufacturer's protocol. Reverse transcription and target gene amplification assays were performed as described previously [18]. The primers used for PCR were shown in Supplementary Table 2.

\section{Statistical analysis}

The results were reported as mean \pm standard deviation (SD), and $P<0.05$ was considered statistically significant. The data were calculated and analyzed by Prism (GraphPad Software Inc., San Diego, CA, USA). Comparisons between controls and treated groups were determined using one-way ANOVA followed by Bonferroni test.

\section{Results}

\section{Bodyweight}

Changes in body weight for the animals from different groups are depicted in Fig. 1A. During the first 3.5 months, 60\% HFD-fed APP/PS1 mice showed significantly higher body weight than ND-fed C57BL/6J and APP/PS1 control mice. When changing the diet to $45 \%$ HFD, we found that the bodyweights of APP/PS1 mice decreased dramatically in comparison with 60\% HFD-fed APP/PS1 mice, especially in mice treated with a low n-6/n-3 PUFA ratio diet; however, the bodyweights of these mice were continuously higher than ND-fed animals.

\section{Serum glucose, insulin levels and lipid profile}

Following $60 \%$ of HFD intervention for 3.5 months, the mice showed a significant increase of fasting blood glucose level and area of under glycemic curve (AUC) than normal diet-fed C57BL/6J and APP/PS1 mice $(P<0.05)$ (Fig. 1B). After changing 60\% HFD to 45\% HFD, a decrease in AUC value was observed in the APP/PS1 mice compared with the 60\% HFD-fed animals, especially in the 45\% HFD and 45\% HFD-N3 diet-fed mice $(P<0.05)$ (Fig. 1D). Moreover, at the end of the experiment, 60\% HFD-fed animals showed a significant increase in serum insulin level in comparison with 45\% HFD-fed animals, and statistical significance was also observed between 60\% HFD, 45\% HFD and 45\% HFD-N6 groups $(P<0.05)$ (Fig. 1E).

The serum lipid profile was presented in Table 2 . We found that $60 \%$ HFD treatment significantly increased serum TC, LDL-C and high-density lipoprotein cholesterol (HDL-C) levels in APP/PS1 mice $(P<$ 0.05). After changing the $60 \%$ HFD diet to $45 \%$ HFD, we observed a significant decrease in serum TC and HDL-c levels, and the mice fed with 45\% HFD-N3 diet showed the lowest serum TC level $(P<0.05)$. Decrease in serum LDL-c level was only observed in 45\% HFD-N3 diet-fed APP/PS1 mice $(P<0.05)$. 
Table 2

Serum lipid levels in mice treated with different diet.

\begin{tabular}{|c|c|c|c|c|c|c|}
\hline \multirow[t]{2}{*}{ Parameters } & C57BL/6J & APP/PS1 & APP/PS1 & APP/PS1 & APP/PS1 & APP/PS1 \\
\hline & ND & ND & $60 \%$ HFD & 45\% HFD & $\begin{array}{l}45 \% \text { HFD- } \\
\text { N3 }\end{array}$ & $\begin{array}{l}45 \% \text { HFD- } \\
\text { N6 }\end{array}$ \\
\hline $\mathrm{TC}(\mathrm{mmol} / \mathrm{l})$ & $\begin{array}{l}2.84 \pm \\
0.46\end{array}$ & $\begin{array}{l}3.76 \pm \\
0.33\end{array}$ & $\begin{array}{l}5.64 \pm \\
0.85^{\mathrm{ab}}\end{array}$ & $\begin{array}{l}4.65 \pm \\
0.47^{\mathrm{abc}}\end{array}$ & $\begin{array}{l}3.67 \pm \\
0.47^{\text {acd }}\end{array}$ & $\begin{array}{l}4.32 \pm \\
0.76^{\mathrm{ac}}\end{array}$ \\
\hline $\mathrm{TG}(\mathrm{mmol} / \mathrm{l})$ & $\begin{array}{l}0.41 \pm \\
0.08\end{array}$ & $\begin{array}{l}0.56 \pm \\
0.09\end{array}$ & $0.38 \pm 0.14$ & $0.50 \pm 0.08$ & $0.58 \pm 0.13^{c}$ & $0.44 \pm 0.06$ \\
\hline $\begin{array}{l}\mathrm{HDL}- \\
\mathrm{c}(\mathrm{mmol} / \mathrm{l})\end{array}$ & $\begin{array}{l}2.13 \pm \\
0.44\end{array}$ & $\begin{array}{l}2.49 \pm \\
0.30\end{array}$ & $\begin{array}{l}3.38 \pm \\
0.60^{\mathrm{ab}}\end{array}$ & $2.97 \pm 0.20^{\mathrm{a}}$ & $2.58 \pm 0.58^{c}$ & $\begin{array}{l}2.88 \pm \\
0.37^{\mathrm{ac}}\end{array}$ \\
\hline $\begin{array}{l}\text { LDL- } \\
\text { c(mmol/l) }\end{array}$ & $\begin{array}{l}0.27 \pm \\
0.03\end{array}$ & $\begin{array}{l}0.34 \pm \\
0.03\end{array}$ & $\begin{array}{l}0.50 \pm \\
0.12^{\mathrm{ab}}\end{array}$ & $0.47 \pm 0.10^{\mathrm{a}}$ & $\begin{array}{l}0.32 \pm \\
0.07^{c d}\end{array}$ & $\begin{array}{l}0.41 \pm \\
0.14^{\mathrm{a}}\end{array}$ \\
\hline
\end{tabular}

Data were expressed as mean $\pm \mathrm{SD}, \mathrm{n}=6$ for each group. a: comparing with C57BL/6J ND group, $P<$ 0.05. b: comparing with APP/PS1 ND group, $P<0.05$. c: comparing with APP/PS1 $60 \%$ HFD group, $P$ $<0.05$. d: comparing with APP/PS1 45\% HFD group, $P<0.05$. HFD: high fat diet; TC: total cholesterol; TG: triglyceride; HDL-c: high density lipoprotein cholesterol; LDL-C: low density lipoprotein cholesterol. C57BL/6J ND: C57BL/6J control mice treated with normal control diet; APP/PS1 ND: APP/PS1 mice treated with normal control diet; APP/PS1 60\% HFD: APP/PS1 mice treated with $60 \%$ high fat diet for 7 months; APP/PS1 HFD-N3: APP/PS1 mice treated with mice $60 \%$ high fat diet for 3.5 months, then $45 \%$ high fat diet with n-3 PUFAs was supplied for another 3.5 months; APP/PS1 45\% HFD: APP/PS1 mice were treated with $60 \%$ high fat diet for 3.5 months, then $45 \%$ high fat diet were provided for another 3.5 months; APP/PS1 45\% HFD-N6: APP/PS1 mice were treated with 60\% high fat diet for 3.5 months, then $45 \%$ high fat diet with n-6 PUFAs supplement were provided for another 3.5 months.

\section{Hepatic histology}

Compared with C57BL/6J control mice, the APP/PS1 control mice showed relatively higher lipid deposition in the livers. At the end of dietary intervention, the mice in the 60\% HFD group showed a significant increase in hepatic lipid deposition. In contrast, hepatic lipid deposition was significantly lessened in mice treated with 45\% HFD animals, especially in the n-3 PUFAs rich diet-treated mice (Fig. 1F).

\section{Inflammatory factors in serum}

As shown in Fig. 1G, 60\% HFD-treated APP/PS1 mice showed higher serum IL-6 and TNF-a levels than ND-fed control mice, and the difference of TNF-a level reached statistical significance compared with control mice $(P<0.05)$. The $45 \%$ HFD decreased serum IL-6 and TNF-a level, and the APP/PS1 mice that received 45\% HFD-N3 diet exhibited the lowest serum TNF-a level $(P<0.05)$, and the difference between 45\% HFD-N3 and 45\% HFD-N6 groups was also significant $(P<0.05)$.

\section{Cortical n-3 and n-6 PUFAs level}

Cortical n-6 and n-3 PUFA levels were shown in Table 3. ND-fed control mice showed lower cortical n-6 PUFAs than $60 \%$ HFD-fed APP/PS1 mice $(P<0.05)$. Compared with $45 \%$ HFD groups, mice from $45 \%$ 
HFD-N6 group showed the highest cortical n-6 PUFAs levels $(P<0.05)$. The $60 \%$ HFD-fed mice showed a significant increase of $n-6 / n-3$ PUFA ratio in the cortex than control mice $(P<0.05)$. Mice from $45 \% \mathrm{HFD}$ and $45 \%$ HFD-N3 groups showed a significant decrease in n-6/n-3 PUFAs ratio the in cortex than mice from $60 \%$ HFD and $45 \%$ HFD-N6 groups $(P<0.05)$

Table 3

The content of cortical fatty acids in mice treated with different diets.

\begin{tabular}{|c|c|c|c|c|c|c|}
\hline Fatty acids & C57BL/6J & APP/PS1 & APP/PS1 & APP/PS1 & APP/PS1 & APP/PS1 \\
\hline $\begin{array}{l}\text { (\% of total cortical fatty } \\
\text { acids) }\end{array}$ & ND & ND & $60 \%$ HFD & 45\% HFD & $\begin{array}{l}45 \% \\
\text { HFD-N3 }\end{array}$ & $\begin{array}{l}45 \% \text { HFD- } \\
\text { N6 }\end{array}$ \\
\hline PUFA & $\begin{array}{l}16.45 \pm \\
1.64\end{array}$ & $\begin{array}{l}17.76 \pm \\
0.10\end{array}$ & $\begin{array}{l}19.39 \pm \\
1.14\end{array}$ & $\begin{array}{l}18.16 \pm \\
1.21\end{array}$ & $\begin{array}{l}19.40 \pm \\
0.37^{\mathrm{a}}\end{array}$ & $\begin{array}{l}19.46 \pm \\
1.74^{\mathrm{a}}\end{array}$ \\
\hline n-6 PUFA & $\begin{array}{l}6.75 \pm \\
0.59\end{array}$ & $\begin{array}{l}6.96 \pm \\
0.03\end{array}$ & $\begin{array}{l}7.88 \pm \\
0.49^{a}\end{array}$ & $\begin{array}{l}7.13 \pm \\
0.39^{c}\end{array}$ & $\begin{array}{l}7.58 \pm \\
0.30^{\mathrm{a}}\end{array}$ & $\begin{array}{l}7.83 \pm \\
0.63^{\text {ad }}\end{array}$ \\
\hline n-3 PUFA & $\begin{array}{l}10.63 \pm \\
1.05\end{array}$ & $\begin{array}{l}10.73 \pm \\
0.06\end{array}$ & $\begin{array}{l}11.34 \pm \\
0.61\end{array}$ & $\begin{array}{l}10.90 \pm \\
0.80\end{array}$ & $\begin{array}{l}11.64 \pm \\
0.25\end{array}$ & $\begin{array}{l}11.45 \pm \\
1.11\end{array}$ \\
\hline DHA & $\begin{array}{l}10.19 \pm \\
1.05\end{array}$ & $\begin{array}{l}10.38 \pm \\
0.08\end{array}$ & $\begin{array}{l}10.95 \pm \\
0.54\end{array}$ & $\begin{array}{l}10.47 \pm \\
0.81\end{array}$ & $\begin{array}{l}11.18 \pm \\
0.26\end{array}$ & $\begin{array}{l}10.99 \pm \\
1.07\end{array}$ \\
\hline EPA & $\begin{array}{l}0.27 \pm \\
0.01\end{array}$ & $\begin{array}{l}0.21 \pm \\
0.02\end{array}$ & $\begin{array}{l}0.23 \pm \\
0.06\end{array}$ & $\begin{array}{l}0.26 \pm \\
0.06\end{array}$ & $\begin{array}{l}0.28 \pm \\
0.03\end{array}$ & $\begin{array}{l}0.29 \pm \\
0.06\end{array}$ \\
\hline$n-6 / n-3$ & $\begin{array}{l}0.63 \pm \\
0.01\end{array}$ & $\begin{array}{l}0.65 \pm \\
0.01\end{array}$ & $\begin{array}{l}0.69 \pm \\
0.01^{\mathrm{ab}}\end{array}$ & $\begin{array}{l}0.65 \pm \\
0.02^{c}\end{array}$ & $\begin{array}{l}0.65 \pm \\
0.03^{c}\end{array}$ & $\begin{array}{l}0.68 \pm \\
0.02^{\text {abde }}\end{array}$ \\
\hline
\end{tabular}

Data were expressed as mean $\pm \mathrm{SD}, n=6$ for each group. ${ }^{\text {a. }}$ comparing with C57 ND group, $P<0.05 .{ }^{\text {b. }}$ : comparing with APP/PS1 ND group, $P<0.05$. c. comparing with APP/PS1 60\% HFD group, $P<0.05$. : comparing with APP/PS1 45\% HFD group, $P<0.05$. e. comparing with APP/PS1 45\% HFD-N3 group, $P$ $<0.05$. HFD: high fat diet; PUFA: polyunsaturated fatty acid; DHA: docosahexaenoic acid; EPA: eicosapentaenoic acid. C57 ND: C57BL/6J control mice treated with normal control diet; APP/PS1 ND: APP/PS1 mice treated with normal control diet; APP/PS1 60\% HFD: APP/PS1 mice treated with $60 \%$ high fat diet for 7 months; APP/PS1 HFD-N3: APP/PS1 mice treated with mice $60 \%$ high fat diet for 3.5 months, then $45 \%$ high fat diet with n-3 PUFAs was supplied for another 3.5 months; APP/PS1 45\% HFD: APP/PS1 mice were treated with $60 \%$ high fat diet for 3.5 months, then $45 \%$ high fat diet were provided for another 3.5 months; APP/PS1 45\% HFD-N6: APP/PS1 mice were treated with 60\% high fat diet for 3.5 months, then $45 \%$ high fat diet with n-6 PUFAs supplement were provided for another 3.5 months.

\section{Behavior}

As showed in Fig. 2A, after 4 days of training, both control mice and HFD-fed APP/PS1 mice showed a significant decrease in escape latency. On day 5, the mice treated with n-3 PUFA rich 45\% HFD showed increased escape latency than mice from other groups, however, we did not observe any statistical significance between the groups $(P>0.05)$. Additionally, the APP/PS1 mice treated with $60 \%$ HFD and $n-3$ PUFA rich diet showed relatively shorter platform crossing times on day 5 than mice from other groups, but the differences between groups are not statistically significant $(P>0.05)$. 


\section{AD pathology}

As shown in Fig. 2B, in comparison with $\mathrm{C} 57 \mathrm{BL} / 6 \mathrm{~J}$ control mice, typical $\mathrm{A} \beta$ plaque deposition was found in the cortexes and hippocampi of APP/PS1 control mice. The treatment of $60 \%$ HFD seemed to have no significant effect on the deposition of $A \beta$ plaque in cortexes and hippocampi of the APP/PS1 mice. Diets with different $n-6 / n-3$ PUFA ratios also had no significant impact on cortical and hippocampal $A \beta$ plaque deposition in the APP/PS1 mice.

As shown in Fig. 2C, treatment of $60 \%$ HFD caused a significant increase of soluble $A \beta_{1-40}$ and $A \beta_{1-42}$ contents in the cortex $(P<0.05)$. The mice treated with n-3 and n-6 PUFAs rich 45\% HFD showed lower cortical soluble $A \beta_{1-40}$ and $A \beta_{1-42}$ content than mice-fed with $60 \%$ HFD $(P<0.05)$. We also found that diets with different n-6/n-3 PUFAs ratio showed discrepant effects on cortical soluble $A \beta_{1-40}$ and $A \beta_{1-42}$ contents, which was indicated by a much lower cortical $A \beta_{1-40}$ and $A \beta_{1-42}$ contents in mice that received n-6 PUFAs rich 45\% HFD than mice treated with n-3 PUFAs rich 45\% HFD $(P<0.05)$. The 60\% HFD-fed mice showed lower cortical aggregated $A \beta_{1-40}$ and $A \beta_{1-42}$ than control mice $(P<0.05)$. The $45 \%$ HFD treatment increased cortical aggregated $A \beta_{1-40}$ and $A \beta_{1-42}$ content dramatically in comparison with mice from control and $60 \%$ HFD groups $(P<0.05)$. The $n-3$ and n-6 PUFAs rich $45 \%$ HFD significantly reduced cortical aggregated $A \beta_{1-40}$ and $A \beta_{1-42}$ content, and the n-6 PUFAs rich 45\% HFD showed the strongest impact on reducing cortical aggregated $A \beta_{1-40}$ and $A \beta_{1-42}$ content $(P<0.05)$. We also found that the mice treated with $n-3$ and $n-6$ PUFAs rich $45 \%$ HFD exhibited a lower aggregated $A \beta_{1-40}$ to $A \beta_{1-42}$ ratio than mice from other treatment groups $(P<0.05)$.

Cortical LDL-C and TC levels are shown in Fig. 2D. Compared with the control mice, the mice fed with $60 \%$ HFD showed a significant increase of cortical LDL-c content $(P<0.05)$. In contrast, $45 \%$ HFD-fed mice showed lower cortical LDL-c levels as compared with mice from $60 \%$ HFD-fed group, and the n-6 PUFAs rich 45\% HFD showed the lowest cortical LDL-c content than mice in other HFD-fed groups $(P<0.05)$. HFD treatment did not affect cortical TC levels, and no statistical significance was observed between the groups $(P>0.05)$.

Cortical amyloid precursor protein (APP) and $\beta$-secretase-1 (BACE1) protein expression were shown in Fig. 2E. APP/PS1 control mice showed higher cortical APP protein expression than C57BL/6J control mice. The treatment of $60 \%$ HFD further up-regulated the cortical APP protein expression in APP/PS1 mice. After changing 60\% HFD diet to $45 \%$ HFD diet, the decreased APP protein expression was observed in the cerebral cortex of APP/PS1 mice, especially in the mice from 45\% HFD and 45\% HFD-N6 groups. The dietary intervention did not affect cortical BACE1 protein expression, and no difference was observed between the groups $(P>0.05)$.

\section{GLUT3 and insulin metabolism-related molecular in the cortex}


Cortical GLUT3 protein expression was shown in Fig. 3A. APP/PS1 control mice displayed lower cortical GLUT3 protein expression in C57BL/6J control mice. The treatment of 60\% HFD significantly increased the cortical GLUT3 protein expression in APP/PS1 mice. After replacing 60\% HFD with 45\% HFD, cortical GLUT3 protein expression was down-regulated and the mice from n-3 PUFAs rich 45\%HFD group showed relatively lower cortical GLUT3 expression than mice treated with 45\% HFD and n-6 PUFAs rich 45\% HFD.

Cortical insulin level was shown in Fig. 3B. The APP/PS1 control mice showed similar cortical insulin level as the C57BL/6J control mice and the treatment of HFDs did not affect cortical insulin level in APP/PS1 mice, although the mice from the $45 \%$ HFD group showed decreases in cortical insulin levels, no statistical significance was observed between the groups $(P>0.05)$.

High-fat diet treatment did not affect cortical insulin receptor (IR), insulin receptor substrate 1 (IRS-1), insulin degrading enzyme (IDE) and phosphorylated-insulin receptors ( $\mathrm{p}$-IR) protein expressions in APP/PS1 mice. The n-6 and n-3 rich 45\% HFD-fed mice showed a significant decrease of cortical p-IR/IR ratio, and the mice fed with n-6 PUFA rich 45\% HFD showed the lowest cortical p-IR/IR ratio than mice from the other groups $(P<0.05)$ (Fig. 3C \& 3D).

As shown in Fig. 3E, APP/PS1 control mice showed relatively higher cortical glycogen synthase kinase-3 $\beta$ (GSK-3 $\beta$ ), but lower $p-G S K 3 \beta / G S K-3 \beta$ ratio than C57BL/6J control mice. The $60 \%$ HFD treatment caused a decrease of cortical phosphorylated-glycogen synthase kinase-3 3 ( $p$-GSK3 $\beta$ ) protein expression in APP/PS1 mice, but no effect on cortical GSK3 $\beta$ protein expression. The $n-6$ and $n-3$ rich $45 \%$ HFD caused a significant decrease of cortical GSK3 $\beta$ protein expression, and the lowest $p$-GSK3 $\beta$ protein expression was observed in $\mathrm{n}-6$ rich $45 \%$ HFD-fed mice $(P<0.05)$. The ratio of $\mathrm{p}-\mathrm{GSK} 3 \beta$ to GSK3 $\beta$ showed a decreasing trend in HFD-treated animals, and the mice from the 45\% HFD-N6 group exhibited the lowest cortical $p$-GSK3 $\beta /$ GSK-3 $\beta$ ratio $(P<0.05)$.

\section{Expression of cortical NF-KB p65 and the mRNA of inflammatory cytokines}

The expression of cortical NF-KB p65 protein expression was shown in Fig. 4A. The red fluorescence was observed in the cortices of all mice groups, indicating that NF-KB was abundantly expressed in the cortex. Meanwhile, mice from 60\% HFD-fed group and n-6 PUFAs rich 45\% HFD-fed group exhibited higher NF-KB p65 expression in the nuclei than mice in other groups, indicating a migration of NF-KB p65 from the cytoplasm to nuclei.

We further examined the mRNA expression of pro-inflammatory cytokines of the NF-KB downstream pathway. As illustrated in Fig. 4B, the APP/PS1 control mice displayed higher cortical mRNA expression of TNF-a, COX2, IL- 6 and IL-1 $\beta$, but lower iNOS mRNA than C57BL/ $6 \mathrm{~J}$ control mice $(P<0.05)$. Compared with APP/PS1 control group, the expression of TNF- $a, C O X 2$, and IL- 6 were down-regulated in APP/PS1 mice receiving either $60 \%$ HFD or $45 \%$ HFD intervention $(P<0.05)$. The same alterations were observed in 
n-6 PUFAs rich 45\% HFD group and n-3 PUFAs rich 45\% HFD group $(P<0.05)$. In addition, we found that n3 PUFAs rich $45 \%$ HFD could potentially elevate the mRNA expression of IL-1 $\beta$ in APP/PS1 mice compared to the C57BL/6J control group, the APP/PS1 60\% HFD-fed group and APP/PS1 45\% HFD-fed group $(P<0.05)$. N-6 rich 45\% HFD caused a significant decrease of cortical IL-1 $\beta$ mRNA expression compared to $45 \%$ HFD-N3 group $(P<0.05)$.

\section{Discussion}

In the current study, AD model mice (APP/PS1) were treated with a HFD to induce insulin resistance and the effect of diet with different n-6/n-3 PUFAs rations on insulin resistance phenotype and AD-like pathology was explored. Our data showed that HFD efficiently induced insulin resistance in APP/PS1 mice, which was indicated by the dramatic increase in body weight, blood glucose, lipids and insulin levels, glucose tolerance, and severe hepatic lipidosis as compared with the normal diet-fed control mice. We also found that a reduction of fat-derived energy (as demonstrated with a 45\% HFD) was efficient in reversing the insulin resistant phenotype that was initially caused by the $60 \%$ HFD in APP/PS1 mice.

Dietary n-6/n-3 PUFA ratio-dependent regulatory effect of HFD on insulin resistance has been previously described, in which the author reported that rats fed with HFD containing n-3 PUFAs ( $n-6 / n-3=1: 1$ ) showed significantly higher insulin sensitivity than rats treated with high n-6 PUFA HFD (n-6/n-3 = 4:1) [19], suggesting that an increase in dietary n-3 PUFAs proportion could improve insulin sensitivity and prevent HFD-induced insulin resistance. It has also been postulated that the visceral adipose tissue (e.g., fatty liver) induced increased circulating free fatty acids (FFAs) and triglycerides, released a significant amount of pro-inflammatory cytokines, and these cytokines disrupt the insulin action, consequently leading to decreased insulin sensitivity and resistance [20]. Consistent with these studies, our data indicated that diets with different n-6/n-3 PUFAs rations discrepantly affected insulin resistance phenotype in $A D$ model mice, which was demonstrated by the significant decrease in body weight, serum TC, LDL-c levels and hepatic lipid deposition in the initial HFD-induced APP/PS1 mice later treated with high n-3 PUFA diet $(n-6 / n-3=1: 1)$. These results align well with the previous report, which showed that $n-6$ PUFA rich diet-fed animals were more likely to manifest liver steatosis [21], while n-3 PUFAs intervention significantly reduced serum TC and LDL-c levels [22], and significantly relieved the signs of HFD-mediated hepatic steatosis. Unexpectedly, in our study, we did not observe the different reflection of serum glucose and insulin levels to diets with different n-6/n-3 PUFA ratios, demonstrating that the regulation of lipids metabolism may partly account for the discrepant insulin resistance relieving effect of the high n-3 PUFA diet $(n-6 / n-3=1: 1)$ in $A D$ model mice.

A study has shown that an HFD resulted in a significant increase in plasma IL- 6 and TNF-a levels in APP/PS1 mice [23]. Consistent with this report, we observed significantly increased levels of serum IL-6 and TNF-a in $60 \%$ HFD-fed mice, and the elevation of serum pro-inflammatory markers were dramatically lowered following treatment with 45\% HFD. Moreover, dietary n-6/n-3 ratio-dependent inhibition of serum TNF-a level was also observed, which was indicated by the lowest serum TNF-a level in n-3 PUFA rich dietfed APP/PS1 mice. High dietary n-6/n-3 PUFAs ratio has been reported to correlate with elevated plasma 
concentration of IL-6 and TNF-a in rats [24]. High n-6/n-3 PUFAs ratio (4:1) HFD-fed SD rats also displayed higher serum IL-6 and TNF- $a$ than low n-6/n-3 ratio (1:1) HFD-fed animals [19]. Reduced IL-6 and TNF-a gene expression have been reported in skeletal muscle tissue of diabetic rat-supplemented with n-3 PUFA [25]. All these results suggested that n-3 PUFA enriched diet might antagonize HFD-induced pro-inflammatory response, and dietary n-6/n-3 PUFAs ratio might have potent modulatory effects on inflammatory reaction in experimental animals.

Reduced n-3 PUFA levels have been found in the brain (mainly in the hippocampus) of AD patients, and the increase of $n-6 / n-3$ PUFAs ratio in the brain has been considered to be associated with the increased risk of AD [26]. In the current study, we did not observe a significant difference in cortical PUFA between C57BL/6J control mice and APP/PS1 control mice, indicating that the mutation of APP gene did not alter the cortical PUFAs profile in the AD model mice. Of note, the treatment of HFD caused an increased trend in cortical PUFAs profile in APP/PS1 mice, although the difference was not statistically significant when compared with control mice. Importantly, our data indicated the potential effect of n-6 PUFA rich diet on elevating cortical n-6 PUFA level and n-6/n-3 PUFAs ratio in APP/PS1 mice, Moreover, this increase of cortical n-6 PUFA level and n-6/n-3 PUFAs ratio effect could be antagonized by $45 \%$ HFD ( $n-6 / n-3=5: 1)$ and 45\% HFD-N3 $(n-6 / n-3=1: 1)$ diets. All these results indicated a strong correlation between cerebral PUFAs profile and dietary fatty acids status, indicating a potential modifying role of dietary n-6/n-3 PUFAs ratio on cerebral n-6 and n-3 PUFA levels.

The senile plaque remains the pathological hallmark sign in AD process, and results from clinical studies have also shown that $n-3$ PUFA interventions can alleviate AD pathological changes and prevent synaptic degeneration [27]. Data from experimental animals also found that n-3 PUFAs rich diet has been effective in inhibiting the formation of amyloid plaque in the hippocampus and cortex in APP transgenic mice [28]. In our study, although the number of plaques in 45\% HFD-N3 diet-fed APP/PS1 mice showed a slightly lesser number of senile plaques than mice treated with control diet, 60\% HFD and 45\% HFD-N6 diet, no statistical significance was observed between groups. We further detected cortical soluble and insoluble $A \beta_{1-40}$ and $A \beta_{1-42}$ levels. Consistent with a previous report [29], we found that HFD treatment increased cortical soluble $A \beta_{1-40}$ and $A \beta_{1-42}$ levels in APP/PS1 mice, indicating that HFD might promote the generation of neurotoxic soluble $A \beta_{1-42}$ and potentially accelerate synaptic degeneration and $A D$ pathological process. It was reported that the excessive increase of lipids (especially cholesterol) in the brain could modify the composition and function of lipid raft in neuronal membranes and enhance $\beta$ secretase-mediated APP processing, ultimately promoting over-generation of $A \beta$ [30]. Thus, we further examined cortical cholesterol levels in mice treated with different diets. The dramatic increase in cortical LDL-c was observed in 60\% HFD-fed APP/PS1 mice, but HFD fortified with PUFAs significantly reduced cortical LDL-c levels, especially in HFD-N6 diet-fed group. This finding is consistent with a previous study, that identified n-6 PUFA as a potent down-regulator of LDL-c level and alleviating depressed LDL receptor activity while promoting the diminishment of circulating LDL-c [31]. We, therefore, speculated that the disturbance of cerebral lipid hemostasis in HFD-fed animals might contribute to the increased cortical soluble $A \beta$ content observed in the AD model mice. Ettcheto et al. demonstrated that HFD-fed APP/PS1 
mice showed significantly higher cerebral insoluble $A \beta_{1-42}$ content than ND-fed control animals [32]. On the contrary, we found that $60 \%$ HFD treatment decreased cortical insoluble $A \beta_{1-40}$ and $A \beta_{1-42}$ levels. $A$ study conducted in FAT-1 mice has found that n-3 PUFA enriched diet could promote the clearance of $A \beta$ from the brain through the cerebral lymphatic system [33]. In our study, we observed an n-6/n-3 ratiodependent effect of HFD on regulating cortical soluble and insoluble $A \beta$ levels, which was demonstrated by lower soluble and insoluble A $\beta$ levels in mice treated with 45\% HFD-N3 and 45\% HFD-N6 diets. Also, consistent with the change in LDL-c level as documented earlier, we found that the diet with the highest dietary $n-6 / n-3$ ratio caused the most significant decrease in soluble and insoluble $A \beta$ levels in the cortex, suggesting the important role of $n-6 / n-3$ PUFA ratio in modulating cerebral $A \beta_{1-40}$ and $A \beta_{1-42}$ levels. Our findings also imply that specific PUFAs ( $n-3$ or $n-6)$ may be critically required by cerebral components to execute optimal brain function(s) and that could account for the observed discrepant levels of cortical $A \beta$ and LDL-c in response to HFD fortified with high n-6/n-3 PUFAs ratio. Further studies are needed to uncover the underlying mechanisms behind the observed discrepant response of $A \beta$ levels to dietary $n$ 6/n-3 PUFA ratio.

It is well known that BACE1 catalyzes the formation of the amyloid-beta peptide from the APP. An increase in BACE1 activity enhanced the production of $A \beta$ and triggered the deposition of $A \beta$ plaque in the brain [34]. In our study, we only observed a decreased trend of APP in mice treated with different $n-6 / n-3$ PUFA ratios without affecting the protein expression of BACE1. This finding suggests that the APP was modulated by PUFAs in HFD, and this modulatory effect was exclusive to BACE1 gene activity. The mismatched changes in APP and BACE1 protein expressions and cerebral A $\beta$ content suggest the involvement of other potential molecular mechanisms in the homeostasis of $A \beta$ in the brain.

The IR is a kind of receptor from the tyrosine kinase family. After binding with insulin, IR activates intracellular tyrosine kinase and initiates intracellular signaling pathways involving a series of physiological responses. Reduced IR sensitivity and hyperphosphorylation of IR have been reported in AD patients [35], potentially suggesting that impaired insulin responsiveness in the brain may be intrinsic to AD pathogenesis. In the current study, we found that the mice treated with n-3 and n-6 PUFA rich diets showed a significant decrease in cortical $p$-IR/IR ratio. The decrease of $p-I R / I R$ ratio indicates the inhibition of insulin metabolic pathway, which in turn inhibits the glucose uptake. Vishal Kothari et al. also reported that the HFD-fed C57BL/6J mice exhibited a lower p-IR/IR ratio than the normal diet-fed control animals [36]. Contrary to that finding, in our study, $60 \%$ and $45 \%$ HFD did not cause a significant decrease of cortical p-IR/IR ratio, however, a decrease of cortical p-IR/IR ratio was observed, especially in mice treated with n-6 PUFA rich 45\% HFD, suggesting that dietary PUFAs have the potential in regulating phosphorylation of IR in HFD-fed APP/PS1 mice. It has been reported that increasing pro-inflammatory cytokines (such as TNF- $a$ and IL-6) can potentially disorient insulin metabolic action(s) and receptor functioning [37]. Excessively high n-6 PUFAs was reported to accelerate oxidative stress and oxidized LDL-c [38]. All, these results could partially explain the decrease of cortical p-IR/IR ratio which was especially observed in mice treated with n-6 PUFA rich 45\% HFD. 
Insulin mRNA expression has been found in both human and mouse brain samples. Rhea et al. also reported that insulin can transport across the blood-brain barrier (BBB) in vivo with either loss or inhibition of the signaling-related insulin receptor, but the binding of insulin to the brain endothelial cells would be decreased [39]. In our study, APP/PS1 mice showed similar cortical insulin levels with C57BL/6J control mice, indicating that the AD pathological changes did not affect the insulin content in the AD model mice. Also, we did not observe any alteration in cortical insulin level in mice treated with HFD. Stanley et al. reported that peripheral hyperinsulinemia could not affect cerebral insulin signaling and insulin levels [40]. Moreover, a study has proved that cells from the hippocampus and olfactory bulb can produce insulin [41]. Our study further confirms that the cerebral insulin homeostasis cannot be affected by peripheral hyperinsulinemia in APP/PS1 mice, and we reasonably hypothesized that this restriction to the hyper-permeability of insulin to the cortex may be tightly regulated by the BBB. The inconsistent changes of serum and cortical insulin level in response to HFD treatment indicated a discrepant regulating mechanism of peripheral and central insulin metabolism. Further investigations are needed to elucidate the underlying mechanism attributing to the discrepant peripheral and central insulin metabolism.

Glucose uptake in the brain is dependent on IR-stimulated translocation of glucose transporter in the plasma membrane. GLUT3 is extensively expressed in the cortex and plays an important role in neuronal glucose uptake. Evidence suggests that HFD has the potential of reducing cerebral GLUT3 protein expression and causing impaired cerebral glucose uptake [36]. Conversely, Kothari and colleagues' study reported a compensatory overexpression of GLUT3 in the brains of diabetic rats [42]. In the present study, APP/PS1 mice showed lower cortical expression of GLUT3 protein than C57BL/6J control mice, which indicated an AD-like pathology-mediated decrease of cerebral glucose uptake in the AD-model mouse. We also found that $60 \%$ HFD-fed APP/PS1 mice displayed a significant increase of cortical GLUT3 expression, suggesting that HFD was efficient in reversing the downregulated glucose uptake in the brain in AD model mice. These results indicated that the increased cortical expression of GLUT3 protein expression could be a compensatory genetic positive-feed response to the insulin resistance initially induced by the HFD, and potentially due to a decreased threshold to cortical insulin sensitivity (since the primary energy source to the brain is obtained from glucose). Prolonged exposure to HFD not only induces pro-inflammatory cytokines and insulin resistance but may also be involved in adaptive genetic feedback response to engage essential proteins and transporters to compensate and improve glucose or nutrient uptake into cells as we have observed with GLUT3 in these HFD-induced insulin resistant APP/PS1 mice. Relative lower cortical GLUT3 expression was found in APP/PS1 mice from 45\% HFD-N3 group as compared with mice treated with 45\% HFD and 45\% HFD-N6 diets, indicating a dietary PUFAsdependent optimal response of cerebral GLUT3 expression. On the contrary, we detected higher GLUT3 expression in the liver and GLUT4 expression in muscle in APP/PS1 control mice in comparison with C57BL/6J mice (Supplementary Fig. 2A\&B). This observation suggests that there may be an overlap of peripheral (hepatic and skeletal) insulin resistance phenotype and AD-like pathological cascade in these ND-fed APP/PS1 mice, implying that AD pathologic process (particularly, APP gene dysfunction) may be a risk factor for peripheral insulin resistance. Further study is required to ascertain this observation. We, however, observed a significant decrease of these GLUTs in 60\% HFD-fed APP/PS1 mice in liver and 
muscle tissues (potentially due to a slightly increased threshold to peripheral insulin sensitivity) further indicating a discrepant peripheral and central glucose metabolism in response to HFD intervention in APP/PS1 mice. Additionally, the divergent sensitivities of liver or muscle GLUT3/4 expression in mice treated with the different diets also hint the role of dietary PUFA rations on glucose uptake in peripheral tissues.

IRSs are known to be major substrates of receptor kinases, mediating insulin-like growth factors (IGFs)/insulin signals to direct anabolic bioactivities, such as energy and glucose homeostasis, growth and adipocyte differentiation [43]. Postmortem studies have described a reduction in brain expression of IR adapter protein IRS-1 in AD subjects [44]. In comparison with C57BL/6J mice, significantly upregulated IRS-1 protein expression was found in the hippocampus of APP/PS1 mice [45]. The IDE is responsible for insulin breakdown in various tissues. Results from other studies have indicated that HFD-fed APP/PS1 mice showed significantly lower brain IDE protein expression than normal diet-fed control animals [46]. We found that the IRS-1 and IDE cortical protein expression remained unchanged in APP/PS1 mice treated with different diets. These results indicate that the effect of $n-6 / n-3$ PUFA ratios in HFD on cerebral glucose homeostasis and insulin signaling pathway in APP/PS1 mice are not entirely attributable to the regulation of IRS-1 and IDE expression. The other mechanisms, such as the regulation of enzyme activity, interactions or binding between substrates and enzymes, might contribute to the HFDmediated changes of central insulin metabolism, warranting further exploration.

The GSK3 $\beta$ is a key molecule involving in neural progenitor cell proliferation, neuronal polarity, and neuroplasticity [47]. Insulin could stimulate phosphorylation of GSK3 $\beta$ and consequently result in bidirectional (phosphorylation and inactivation) effect on glycogen synthase, an enzyme responsible for glycogenesis. Elevated total GSK3 $\beta$ protein expression indicates hyper-activity of GSK3 $\beta$ signaling [48]. In the pathology of $A D, G S K 3 \beta$ has been shown to promote Tau phosphorylation through the insulin/phosphoinositide 3-kinase (PI3K)/protein kinase B (Akt) signaling pathway [49]. In our study, APP/PS1 mice showed a relatively higher cortical GSK3 $\beta$ expression but lower $p$-GSK3 $\beta / G S K 3 \beta$ ratio than $\mathrm{C} 57 \mathrm{BL} / 6 \mathrm{~J}$ control mice, indicating a potential activation of the GSK3 $\beta$ signaling pathway in this $A D$ model mice. HFD treatment has been reported to cause a decrease in cortical p-GSK3 $\beta$ expression and pGSK3 $\beta /$ GSK3 $\beta$ ratio in APP/PS1 mice, suggesting a regulatory effect of HFD on the phosphorylation of GSK3 $\beta$ and in modifying the activity of GSK3 $\beta$. Moreover, a decreased trend of cortical GSK3 $\beta$ expression was found in 45\% HFD-N3 and 45\% HFD-N6 diets treated mice. Additionally, significant decreases of cortical p-GSK3 $\beta$ and $p$-GSK3 $\beta /$ GSK3 $\beta$ ratio were also found in 45\% HFD-N6 diet-fed APP/PS1 mice, indicating hyper-activation of GSK3 $\beta$ in these high n-6/n-3 PUFA ratio diet-fed APP/PS1 mice. Together with the relatively high cortical GLUT3 protein expression observed in these animals, we speculate that diet with a high n-6/n-3 PUFA ratio might affect brain glucose metabolism through regulating the phosphorylation of GSK3 $\beta$, therefore affecting the uptake of glucose in the brain. Altogether, our findings indicated that the discrepant regulation of GSK3 $\beta$ activation might contribute to the dietary $n-6 / n-3$ PUFA ratio-associated modifying effect in insulin signaling pathway in the brain of APP/PS1 mice. 
NF-KB is a nuclear transcription factor that belongs to the Rel protein family, and accumulating evidence suggests its critical role in the pathophysiology of AD. Activated NF-KB p65 in the brain could regulate the expression of many genes, most of which encode immune proteins with pro-inflammatory activities [50]. Consistent with a previous study [51], we detected that cortical NF-KB p65 translocated from the cytoplasm to nuclei in the cortex in mice-treated with $60 \%$ HFD, indicating the activation of NF-KB p65 signaling pathway. While, the treatment of PUFAs rich HFD treatment inhibited the intracellular translocation of NF-KB p65, especially in n-3 PUFAs rich HFD-fed animals. We also found that APP/PS1 mice showed significantly up-regulated cortical TNF-a, COX2, IL-1 $\beta$ and IL-6 mRNA expressions, but lower iNOS mRNA expression than C57BL/6J mice, demonstrating an enhanced neuro-inflammatory response in these AD model mice. Anthony Pinçon et al. reported that administration of a high-cholesterol diet caused 38\% increase of cortical TNF-a mRNA expression in APP/PS1 mice as compared with control mice without affecting IL-6 and IL-1 $\beta$ mRNA expressions [52]. Inconsistent with this report, we observed a significant decrease in cortical TNF-a, COX2 and IL-6 mRNA expression, but an increase in iNOS mRNA expression in HFD-fed APP/PS1 mice. The discrepancy between studies might attribute to the diversity of intervention diet or the study design. Moreover, we observed that the $60 \%$ and $45 \%$ HFDs did not affect cortical IL-1 $\beta$ mRNA expression. In contrast, n-3 PUFAs-rich 45\% HFD significantly increased cortical IL-1 $\beta$ mRNA level, and a decrease expression was observed in mice treated with n-6 PUFAs-rich 45\% HFD.

Although it is commonly recognized that n-6 PUFA has pro-inflammatory functions as prostaglandin and leukotriene precursors, n-6 PUFA can also be converted into anti-inflammatory mediators or modulate the gene expression of the pro-inflammatory cytokine to exert anti-inflammatory effects. Alashmali et al. reported that adequate dietary n-6 PUFA intake restored hippocampal inflammation cytokine gene expression caused by intra-cerebroventricular lipopolysaccharide (LPS) injection [53]. We, therefore, speculate that the AD-like pathology in APP/PS1 mice might have caused hyperactivated neuroinflammation in the brain, which could be antagonized by the treatment with HFD. The inconsistent expressional pattern of pro-inflammatory cytokines in the cortexes of HFD-fed mice further indicated the potentially varied regulatory effect(s) of dietary fatty acids on the cerebral inflammatory response. Thus, further studies are needed to elucidate the relationship between HFD (especially with different PUFAs ratios) and neuroinflammation in modulating $A D$ pathology process.

\section{Limitations}

A few limitations remain in this study. First, due to the young age of the mice and the short duration of the intervention, no significant differences were found in learning and memory abilities. Therefore, a much longer period of dietary intervention was required of to explore the persistent effects of dietary PUFAs on these behavioral indices. Second, we only used male APP/PS1 mice for experiment, while recent studies have shown that APP/PS1 transgenic mice showed a gender difference in behavior and AD-like pathology in brain [54]. Thus, it is essential to further investigate the sex-based effects of dietary PUFAs on APP/PS1 mice. Also, due to the limited brain species, we fall to detect the level of proinflammatory cytokine in the brain, thus, we could not provide a more accurate cerebral neuroinflammatory profile in the experimental animals. 


\section{Conclusion}

In conclusion, HFD induced insulin resistance phenotype in peripheral tissues and increased circulating pro-inflammatory cytokine levels in APP/PS1 mice. Dietary n-6/n-3 PUFA ratio plays a critical role in modifying $A D$ pathological process, insulin signaling pathway, and neuroinflammation in the cortex in response to high-fat diet treatment. In preventing AD, optimal dietary n-6/n-3 PUFAs ratio should be taken into consideration in PUFA-fortified diet interventional strategy. Extensive studies are essentially needed to further uncover the relevant underlying molecular mechanisms and bridge the knowledge gap associated with the potential effects of dietary PUFAs on insulin resistance and cortical degeneration in $\mathrm{AD}$.

\section{Abbreviations}

T2DM

Type 2 diabetes mellitus; AD:Alzheimer's disease; PUFAs:Polyunsaturated fatty acids; HFD:high-fat diet; ND:normal diet; DHA:Docosahexaenoic acid; ARA:arachidonic acid; GTT:glucose tolerance test; AUC:under glycemic curve; NF-кB p65:Nuclear factor kappa-B p65; GLUTs:Glucose transporters; TNFa:Tumor Necrosis Factor-a; IL-1 $\beta$ :Interleukin-1 $\beta$; IL-6:Interleukin-6; COX2:Cyclooxygenase 2; iNOS:inducible nitric oxide synthase; TC:Total cholesterol; LDL-c:Low-density lipoprotein cholesterol; HDL-c:High-density lipoprotein cholesterol; IR:Insulin receptor; IRS-1:insulin receptor substrate 1; IDE:insulin degrading

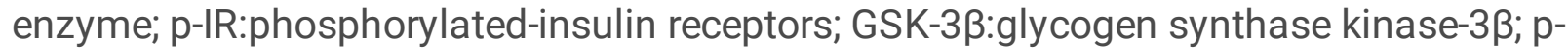

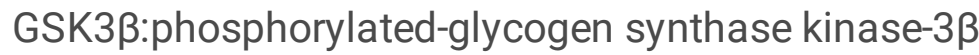

\section{Declarations}

\section{Consent for publication}

Not applicable.

\section{Availability of data and materials}

The datasets used and analyzed during the current study are available from thecorresponding author on reasonable request.

\section{Competing Interest}

The authors declare that they have no competing interests.

\section{Funding}

This study was supported by grants from the National Natural Science Foundation of China (No. 81973027).The funders/sponsors had no role in the design andconduct of the study; analysis, or 
interpretation ofthe data; preparation, review, or approval of the manuscript; and decision tosubmit the manuscript for publication.

\section{Authors'contributors}

Linhong Yuan designed the work; Xiaojun Ma, Yujie Guo, Pengfei Li and Jingjing Xu performed most of the experiments; Shengqi Dong, Pengfei Li and Yanyan Gao carried out other lab works; Linhong Yuan, Nicholas Van Halm-Lutterodt and Xiaojun Ma contributed to the data interpretation and drafting the manuscript. All authors approved the final version of the manuscript.

\section{Acknowledgements}

Not applicable.

\section{References}

1. Reitz, C., C. Brayne, and R. Mayeux, Epidemiology of Alzheimer disease. Nat Rev Neurol, 2011. 7(3): p. 137-52.

2. Talbot, K., et al., Demonstrated brain insulin resistance in Alzheimer's disease patients is associated with IGF-1 resistance, IRS-1 dysregulation, and cognitive decline. J Clin Invest, 2012. 122(4): p. 131638.

3. Razay, G., A. Vreugdenhil, and G. Wilcock, The metabolic syndrome and Alzheimer disease. Arch Neurol, 2007. 64(1): p. 93-6.

4. Janson, J., et al., Increased risk of type 2 diabetes in Alzheimer disease. Diabetes, 2004. 53(2): p. 474-81.

5. Rivera, E.J., et al., Insulin and insulin-like growth factor expression and function deteriorate with progression of Alzheimer's disease: link to brain reductions in acetylcholine. J Alzheimers Dis, 2005. 8(3): p. 247-68.

6. Steen, E., et al., Impaired insulin and insulin-like growth factor expression and signaling mechanisms in Alzheimer's disease-is this type 3 diabetes? J Alzheimers Dis, 2005. 7(1): p. 63-80.

7. Martin Prince, et al., World Alzheimer Report 2014: Dementia and Risk Reduction an Analysis of Protective and Modifiable Factors. 2014.

8. Freund-Levi, Y., et al., Omega-3 supplementation in mild to moderate Alzheimer's disease: effects on neuropsychiatric symptoms. Int J Geriatr Psychiatry, 2008. 23(2): p. 161-9.

9. Cederholm, T., N. Salem, Jr., and J. Palmblad, $\omega-3$ fatty acids in the prevention of cognitive decline in humans. Adv Nutr, 2013. 4(6): p. 672-6.

10. Peet, M., et al., Depletion of omega-3 fatty acid levels in red blood cell membranes of depressive patients. Biol Psychiatry, 1998. 43(5): p. 315-9.

11. Hooper, C., et al., The Relationship of Omega 3 Polyunsaturated Fatty Acids in Red Blood Cell Membranes with Cognitive Function and Brain Structure: A Review Focussed on Alzheimer's Disease. 
J Prev Alzheimers Dis, 2018. 5(1): p. 78-84.

12. Bigornia, S.J., et al., Prospective Associations of Erythrocyte Composition and Dietary Intake of n-3 and n-6 PUFA with Measures of Cognitive Function. Nutrients, 2018. 10(9).

13. Marangoni, F., et al., Dietary linoleic acid and human health: Focus on cardiovascular and cardiometabolic effects. Atherosclerosis, 2020. 292: p. 90-98.

14. Meng Liping, et al., The dietary fatty acids intakes and their food sources among Chinese adults. Acta Nutrimenta Sinica, 2009. 31(05): p. 424-427.

15. Simopoulos, A.P., An Increase in the Omega-6/Omega-3 Fatty Acid Ratio Increases the Risk for Obesity. Nutrients, 2016. 8(3): p. 128.

16. Streijger, F., et al., Structural and behavioural consequences of double deficiency for creatine kinases BCK and UbCKmit. Behav Brain Res, 2005. 157(2): p. 219-34.

17. Folch, J., M. Lees, and G.H. Sloane Stanley, A simple method for the isolation and purification of total lipides from animal tissues. J Biol Chem, 1957. 226(1): p. 497-509.

18. Dong, S., et al., Dietary Vitamin E Status Dictates Oxidative Stress Outcomes by Modulating Effects of Fish Oil Supplementation in Alzheimer Disease Model APP(swe)/PS1(dE9) Mice. Mol Neurobiol, 2018. 55(12): p. 9204-9219.

19. Qiu, Y., et al., [Effects of dietary different ratios of high $n-3 / n-6$ polyunsaturated fatty acids on insulin resistance in rats]. Wei Sheng Yan Jiu, 2013. 42(1): p. 10-3.

20. Tchernof, A. and J.P. Després, Pathophysiology of human visceral obesity: an update. Physiol Rev, 2013. 93(1): p. 359-404.

21. Valenzuela, R. and L.A. Videla, The importance of the long-chain polyunsaturated fatty acid n-6/n-3 ratio in development of non-alcoholic fatty liver associated with obesity. Food Funct, 2011. 2(11): p. 644-8.

22. Go, R.E., et al., Effects of microalgal polyunsaturated fatty acid oil on body weight and lipid accumulation in the liver of C57BL/6 mice fed a high fat diet. J Biomed Res, 2016. 30(3): p. 234-42.

23. Walker, J.M., et al., Reversal of high fat diet-induced obesity improves glucose tolerance, inflammatory response, $\beta$-amyloid accumulation and cognitive decline in the APP/PSEN1 mouse model of Alzheimer's disease. Neurobiol Dis, 2017. 100: p. 87-98.

24. Yang, L.G., et al., Low n-6/n-3 PUFA Ratio Improves Lipid Metabolism, Inflammation, Oxidative Stress and Endothelial Function in Rats Using Plant Oils as n-3 Fatty Acid Source. Lipids, 2016. 51(1): p. 4959.

25. Figueras, M., et al., Effects of eicosapentaenoic acid (EPA) treatment on insulin sensitivity in an animal model of diabetes: improvement of the inflammatory status. Obesity (Silver Spring), 2011. 19(2): p. 362-9.

26. Cooper, J.L., Dietary lipids in the aetiology of Alzheimer's disease: implications for therapy. Drugs Aging, 2003. 20(6): p. 399-418. 
27. Che, $\mathrm{H}$., et al., Comparative study of the effects of phosphatidylcholine rich in DHA and EPA on Alzheimer's disease and the possible mechanisms in CHO-APP/PS1 cells and SAMP8 mice. Food Funct, 2018. 9(1): p. 643-654.

28. Lim, G.P., et al., A diet enriched with the omega-3 fatty acid docosahexaenoic acid reduces amyloid burden in an aged Alzheimer mouse model. J Neurosci, 2005. 25(12): p. 3032-40.

29. Nuzzo, D., et al., Insulin Resistance as Common Molecular Denominator Linking Obesity to Alzheimer's Disease. Curr Alzheimer Res, 2015. 12(8): p. 723-35.

30. Wang, C., et al., The relationship between cholesterol level and Alzheimer's disease-associated APP proteolysis/Aß metabolism. Nutr Neurosci, 2019. 22(7): p. 453-463.

31. Hayes, K.C., Dietary fatty acids, cholesterol, and the lipoprotein profile. Br J Nutr, 2000. 84(4): p. 3979.

32. Ettcheto, M., et al., Evaluation of Neuropathological Effects of a High-Fat Diet in a Presymptomatic Alzheimer's Disease Stage in APP/PS1 Mice. J Alzheimers Dis, 2016. 54(1): p. 233-51.

33. Ren, $\mathrm{H}$., et al., Omega-3 polyunsaturated fatty acids promote amyloid- $\beta$ clearance from the brain through mediating the function of the glymphatic system. Faseb j, 2017. 31(1): p. 282-293.

34. Hampel, H., et al., The $\beta$-Secretase BACE1 in Alzheimer's Disease. Biol Psychiatry, 2021. 89(8): p. 745756.

35. Watson, G.S. and S. Craft, The role of insulin resistance in the pathogenesis of Alzheimer's disease: implications for treatment. CNS Drugs, 2003. 17(1): p. 27-45.

36. Kothari, V., et al., High fat diet induces brain insulin resistance and cognitive impairment in mice. Biochim Biophys Acta Mol Basis Dis, 2017. 1863(2): p. 499-508.

37. Nieto-Vazquez, I., et al., Insulin resistance associated to obesity: the link TNF-alpha. Arch Physiol Biochem, 2008. 114(3): p. 183-94.

38. Bolea, G., et al., Digestive n-6 Lipid Oxidation, a Key Trigger of Vascular Dysfunction and Atherosclerosis in the Western Diet: Protective Effects of Apple Polyphenols. Mol Nutr Food Res, 2021. 65(6): p. e2000487.

39. Rhea, E.M., C. Rask-Madsen, and W.A. Banks, Insulin transport across the blood-brain barrier can occur independently of the insulin receptor. J Physiol, 2018. 596(19): p. 4753-4765.

40. Stanley, M., et al., The Effects of Peripheral and Central High Insulin on Brain Insulin Signaling and Amyloid- $\beta$ in Young and Old APP/PS1 Mice. J Neurosci, 2016. 36(46): p. 11704-11715.

41. Kuwabara, T., et al., Insulin biosynthesis in neuronal progenitors derived from adult hippocampus and the olfactory bulb. EMBO Mol Med, 2011. 3(12): p. 742-54.

42. Boileau, P., et al., Overexpression of GLUT3 placental glucose transporter in diabetic rats. J Clin Invest, 1995. 96(1): p. 309-17.

43. Miki, H., et al., Essential role of insulin receptor substrate 1 (IRS-1) and IRS-2 in adipocyte differentiation. Mol Cell Biol, 2001. 21(7): p. 2521-32. 
44. Kleinridders, A., Deciphering Brain Insulin Receptor and Insulin-Like Growth Factor 1 Receptor Signalling. J Neuroendocrinol, 2016. 28(11).

45. Tanokashira, D., W. Fukuokaya, and A. Taguchi, Involvement of insulin receptor substrates in cognitive impairment and Alzheimer's disease. Neural Regen Res, 2019. 14(8): p. 1330-1334.

46. Petrov, D., et al., High-fat diet-induced deregulation of hippocampal insulin signaling and mitochondrial homeostasis deficiences contribute to Alzheimer disease pathology in rodents. Biochim Biophys Acta, 2015. 1852(9): p. 1687-99.

47. Salcedo-Tello, P., A. Ortiz-Matamoros, and C. Arias, GSK3 Function in the Brain during Development, Neuronal Plasticity, and Neurodegeneration. Int J Alzheimers Dis, 2011. 2011: p. 189728.

48. Beasley, C., et al., Glycogen synthase kinase-3beta immunoreactivity is reduced in the prefrontal cortex in schizophrenia. Neurosci Lett, 2001. 302(2-3): p. 117-20.

49. Zhang, Y., et al., Diabetes mellitus and Alzheimer's disease: GSK-3 $\beta$ as a potential link. Behav Brain Res, 2018. 339: p. 57-65.

50. Sharif, O., et al., Transcriptional profiling of the LPS induced NF-kappaB response in macrophages. BMC Immunol, 2007. 8: p. 1.

51. Zhang, X., et al., High dietary fat induces NADPH oxidase-associated oxidative stress and inflammation in rat cerebral cortex. Exp Neurol, 2005. 191(2): p. 318-25.

52. Pinçon, A., et al., Non-Alcoholic Fatty Liver Disease, and the Underlying Altered Fatty Acid Metabolism, Reveals Brain Hypoperfusion and Contributes to the Cognitive Decline in APP/PS1 Mice. Metabolites, 2019. 9(5).

53. Alashmali, S.M., et al., The effects of $n-6$ polyunsaturated fatty acid deprivation on the inflammatory gene response to lipopolysaccharide in the mouse hippocampus. J Neuroinflammation, 2019. 16(1): p. 237.

54. Mifflin, M.A., et al.,Sex differences in the IntelliCage and the Morris water maze in the APP/PS1 mouse model of amyloidosis. Neurobiol Aging. 2021 May;101:130-140.

\section{Figures}


A

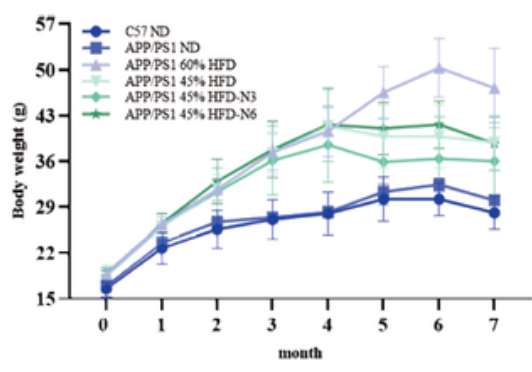

C

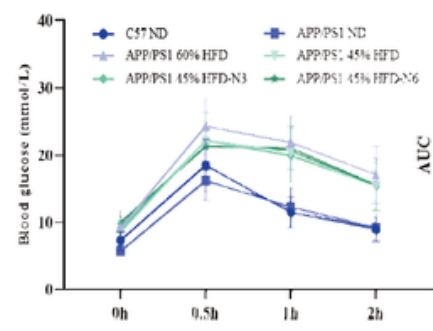

E

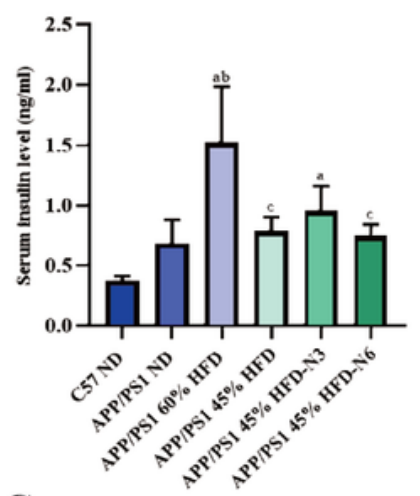

G

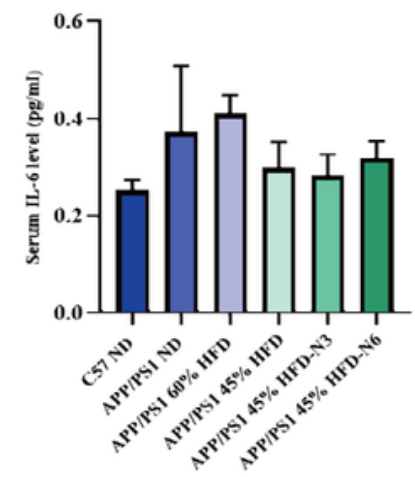

F
B

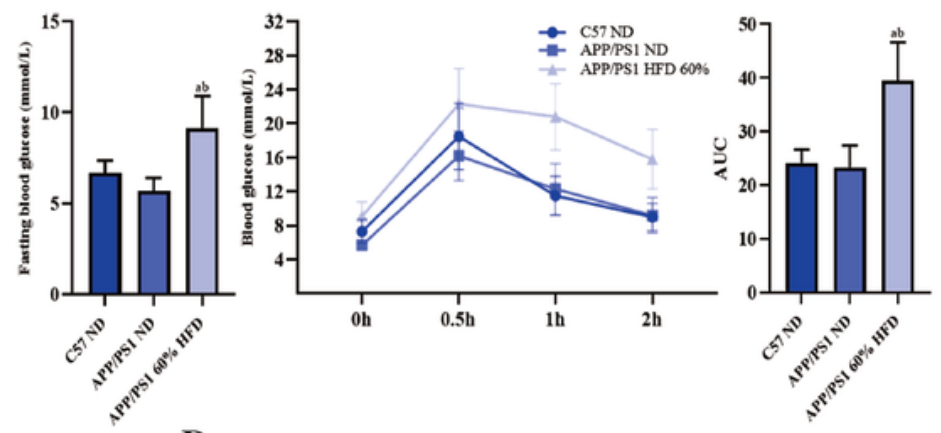

D
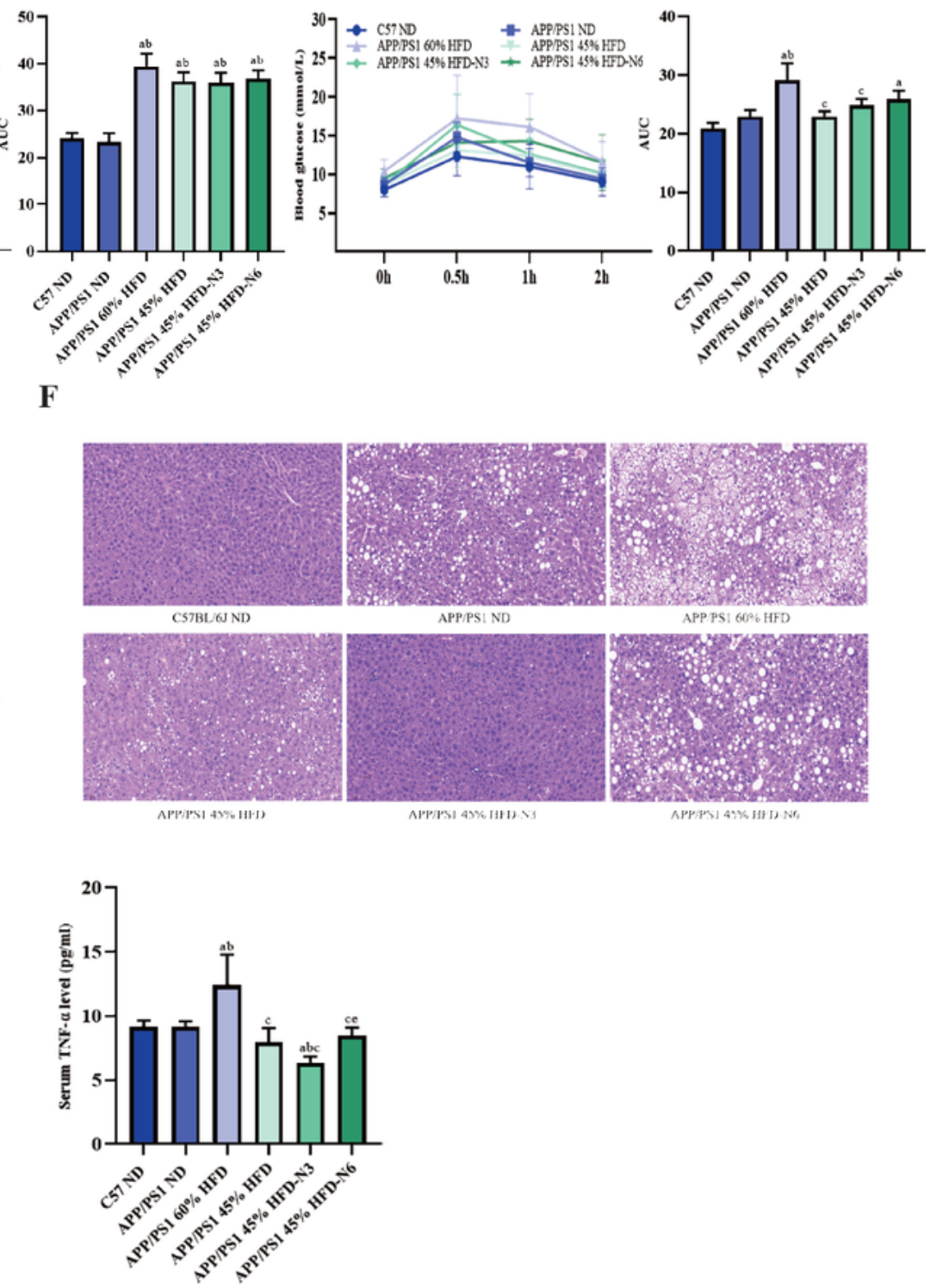

Figure 1

Mice insulin resistancephenotype, hepatic steatosis and peripheral inflammatory cytokine levels.A:Body weight monitoring of C57BL/6J and APP/PS1 mice during the dietary intervention (from the age of 1 month).B: Fastingblood glucose level and glucose tolerancetest (GTT) after HFD intervention for 3.5 months.C: Baseline GTT and area under the curve (AUC) analysis after grouping the animals into different diet-treated groups. D: GTT and AUC analysis at the end of the intervention phase.E: Serum 
insulin level after dietary intervention. F: Histological evaluation of hepatic steatosis in mice treated with different diets. G: Serum IL-6 and TNF-a level in mice treated with different diets.Data were represented as mean $\pm S D, n=6$ in each group. a: comparing with C57BL/6J ND group, $P<0.05$. b: comparing with APP/PS1 ND group, $P<0.05$. c: comparing with APP/PS1 60\% HFD group, $P<0.05$. e: comparing with APP/PS1 45\% HFD-N3 group, $P<0.05$.One-way ANOVA was used for statisticaltestsfollowed by Bonferroni post-test.

A

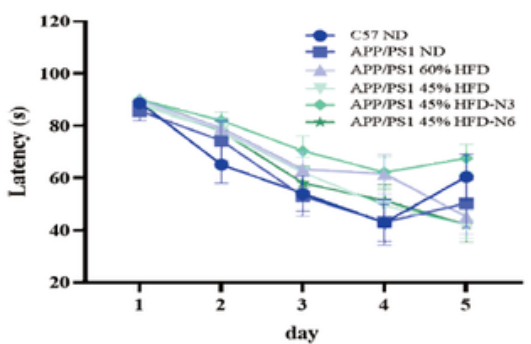

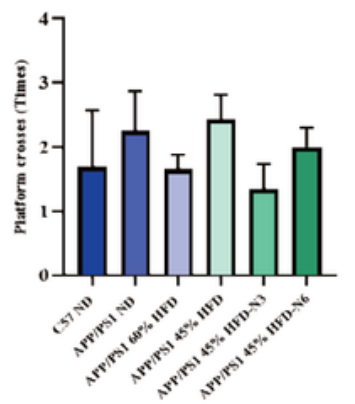

B

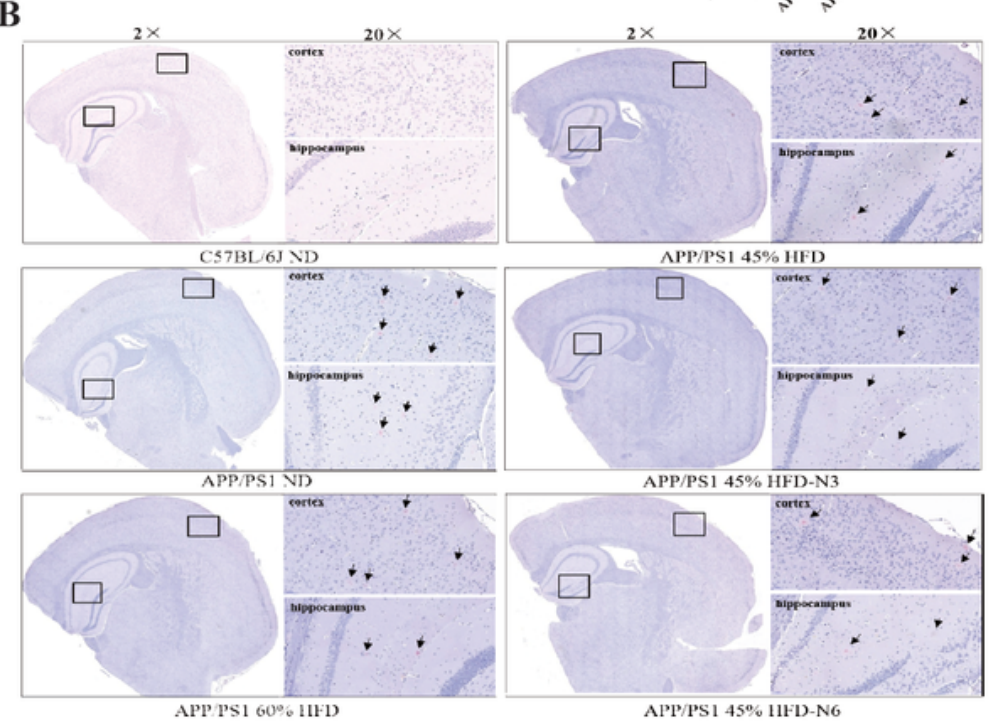

C

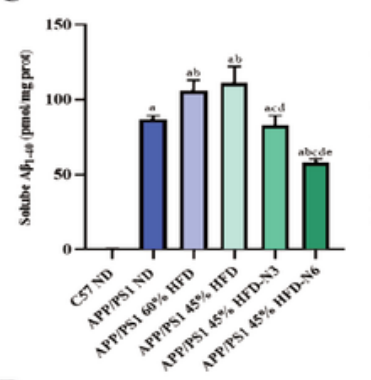

D

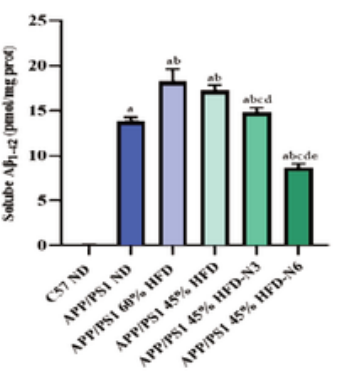

E
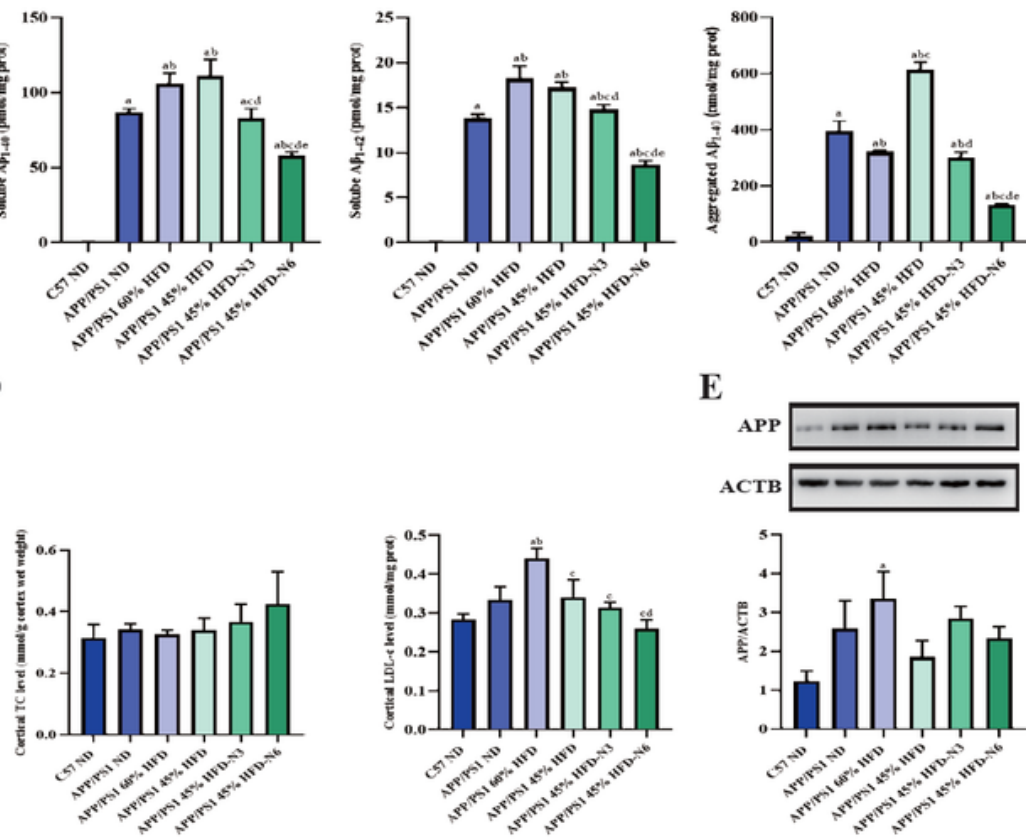
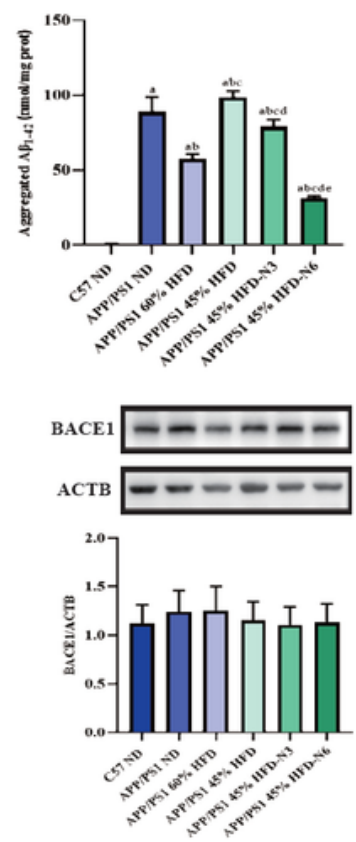

Figure 2 
Behavior and AD pathological changes. A: Results of spatiallearning and memory function after dietary intervention.B: Congo red staining of mice hippocampus and cortex.C: Soluble and Aggregated $A \beta$ in cortex of mice fed with different diets. D: Cortical total cholesterol (TC) and low-density lipoprotein cholesterol (LDL-c) levels of mice fed with different diets.E: Immunoblotsof APP and BACE1 in cortex of mice fed with different diets. Actin was served as the internal control. Data were represented as mean \pm $\mathrm{SD}, \mathrm{n}=6$ in each group. a: comparing with C57BL/6J ND group, $\mathrm{P}<0.05$. b: comparing with APP/PS1 ND group, $\mathrm{P}<0.05$. c: comparing with APP/PS1 60\% HFD group, $\mathrm{P}<0.05$. $\mathrm{d}$ : comparing with APP/PS1 45\% HFD group, $P<0.05$. e: comparing with APP/PS1 45\% HFD-N3 group, $P<0.05$. One-way ANOVA was used for statistical tests followed by Bonferroni post-test. 
A

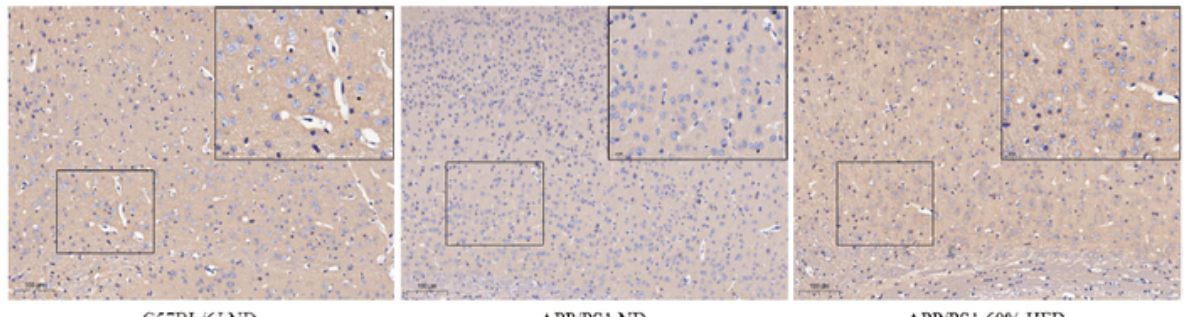

C57BL 6J ND

APP/PS1 ND

APP/PS1 $60 \%$ HFD

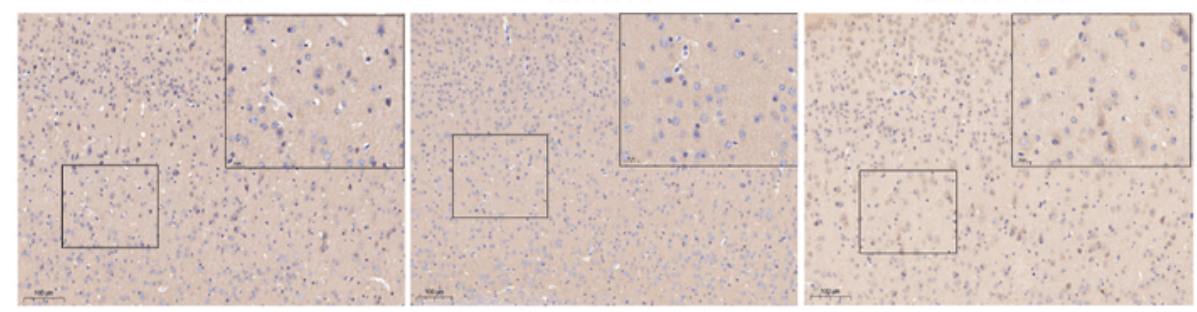

APPPS1 45\% HFD

APP/PS1 45\% HFD-N3

APP/PS1 45\% HFD-N6

B

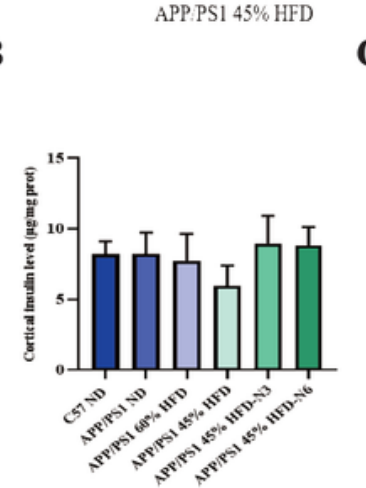

C ${ }_{\text {IR }} \equiv= \pm= \pm=$

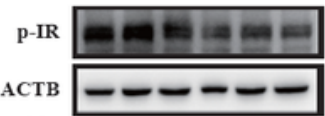
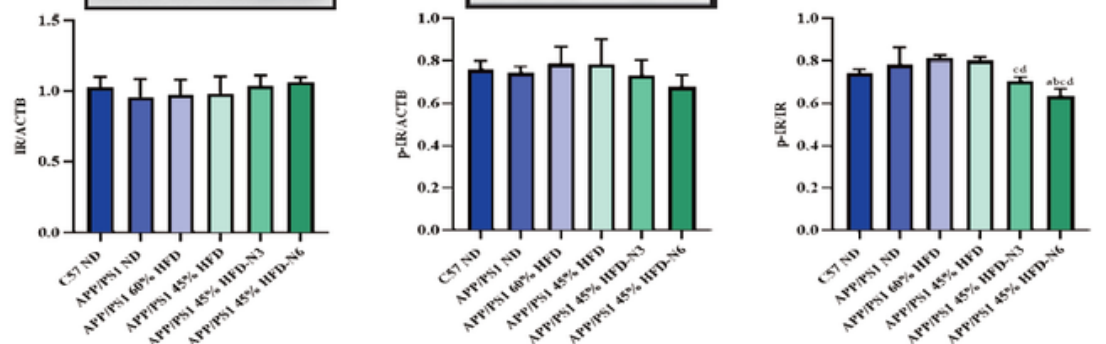

D
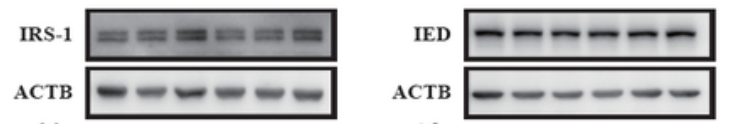

E
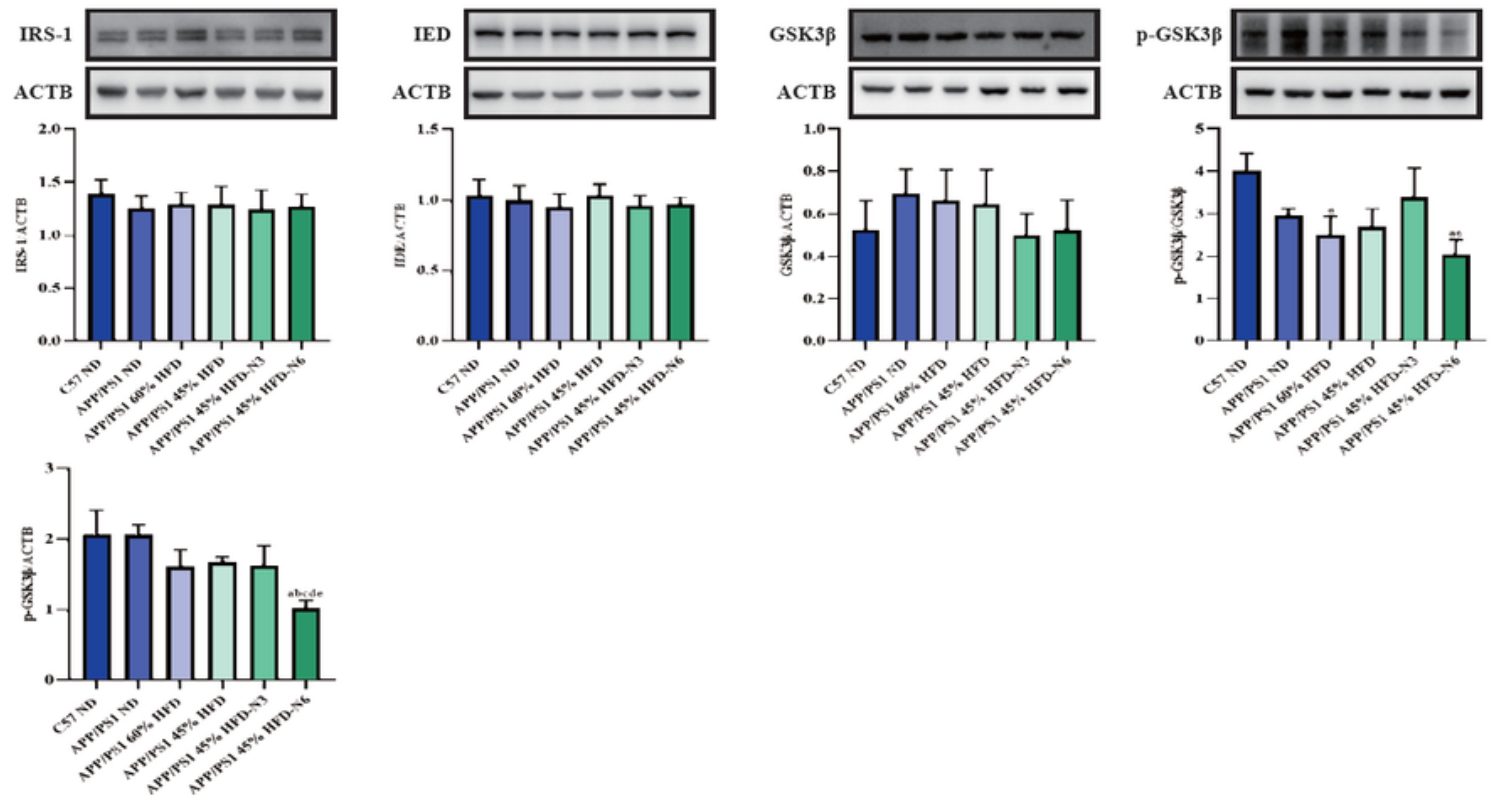

\section{Figure 3}

Glucose transporter and insulin signaling pathway related molecules expression. A:GLUT3 protein expression in cortex of mice fed with different diets. B: Cortical insulin level in mice treated with different diets.C-E:Immunoblots of insulin signaling proteins in cortex of mice fed with different diets. Actinwas served as the internal control.Data were represented as mean $\pm S D, n=6$ in each group. a: comparing with C57BL/6J ND group, $\mathrm{P}<0.05$. b: comparing with APP/PS1 ND group, $\mathrm{P}<0.05$. c: comparing with 
APP/PS1 60\% HFD group, $P<0.05$. d: comparing with APP/PS1 45\% HFD group, $P<0.05$. e: comparing with APP/PS1 45\% HFD-N3 group, P< 0.05. One-way ANOVA was used for statistical tests followed by Bonferroni post-test.

A

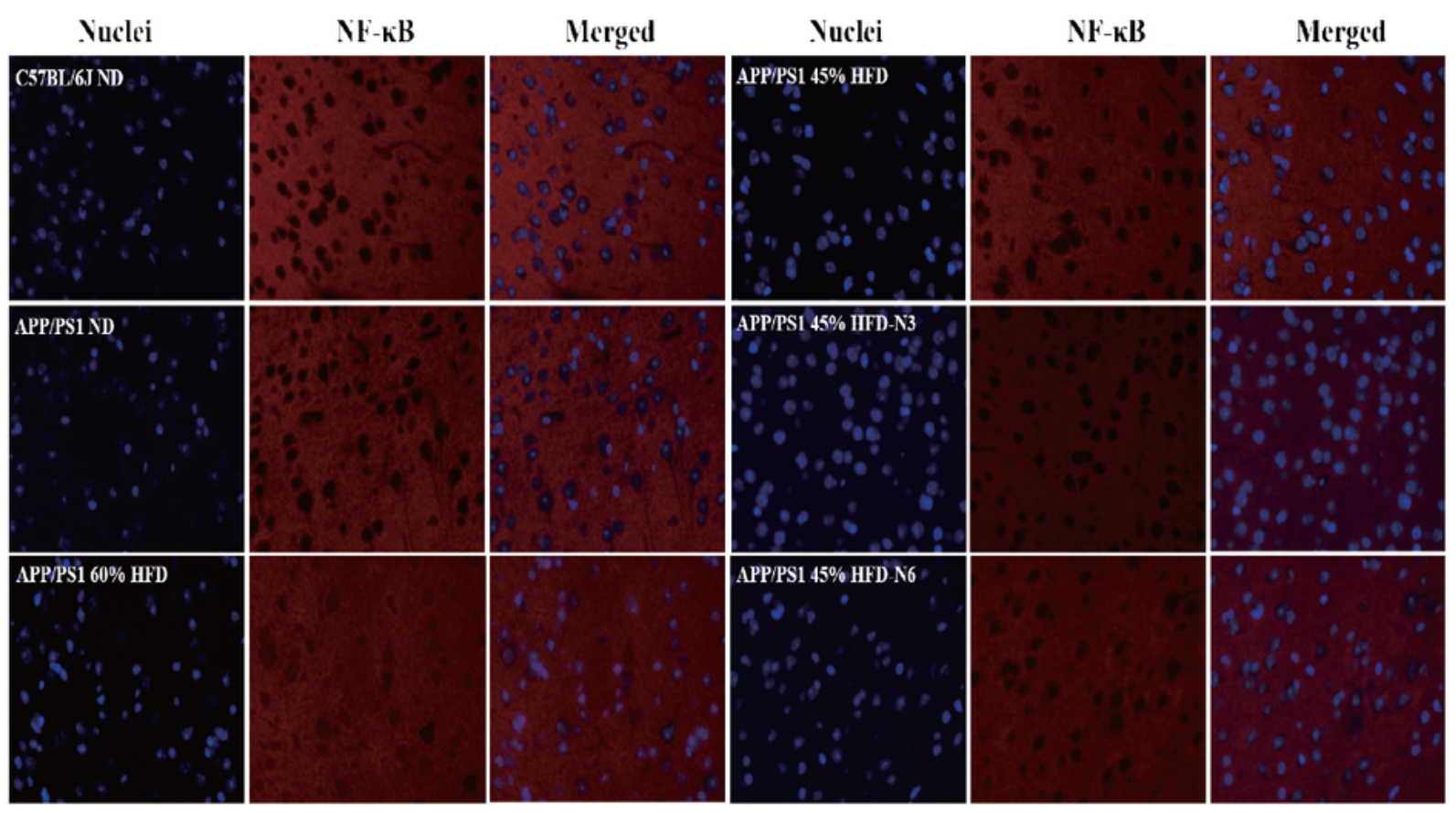

B
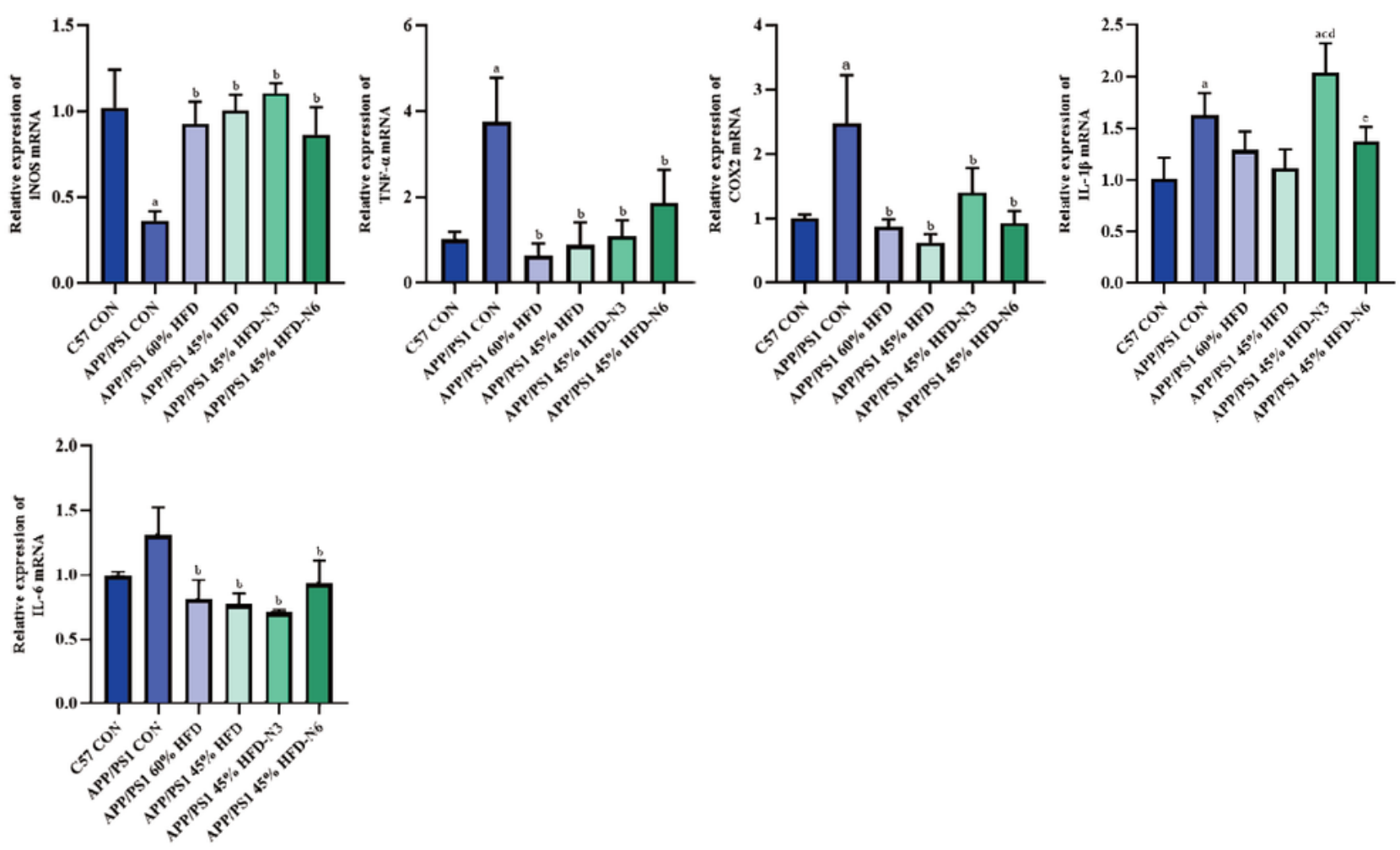

Figure 4

NF-kB p65 expression and its downstream inflammatory gene mRNA expression.A:NF-kBp65 immunofluorescence staining in cortexof mice fed with different diets.B: Cortical mRNA expression levels 
of iNOS, TNF-a,COX2,IL-1 $\beta$ and IL-6.Data were represented as mean \pm SD, $n=6$ in each group. a: comparing with C57BL/6J ND group, $\mathrm{P}<0.05$. b: comparing with APP/PS1 ND group, $\mathrm{P}<0.05$. $\mathrm{C}$ : comparing with APP/PS1 60\% HFD group, $P<0.05$. d: comparing with APP/PS1 45\% HFD group, $P<0.05$. e: comparing with APP/PS1 45\% HFD-N3 group, $P<0.05$. One-way ANOVA was used for statistical tests followed by Bonferroni post-test.

\section{Supplementary Files}

This is a list of supplementary files associated with this preprint. Click to download.

- Supplementarymaterial.pdf 